\title{
A gradient-based uncertainty optimization framework utilizing dimensional adaptive polynomial chaos expansion
}

\author{
Hai Fang ${ }^{l}$, Chunlin Gong ${ }^{l} \bowtie, H u a ~ S u^{l}$, Yunwei Zhang ${ }^{l}$, Chunna Li $i^{l}$, Andrea Da Ronch ${ }^{2}$
}

\begin{abstract}
To improve the efficiency of solving uncertainty design optimization problems, a gradient-based optimization framework is herein proposed, which combines the dimension adaptive polynomial chaos expansion (PCE) and sensitivity analysis. The dimensional adaptive PCE is used to quantify the quantities of interest (e.g. reliability, robustness metrics) and the sensitivity. The dimensional adaptive property is inherited from the dimension adaptive sparse grid, which is used to evaluate the PCE coefficients. Robustness metrics, referred to as statistical moments, and their gradients with respect to design variables are easily derived from the PCE, whereas the evaluation of the reliability and its gradient require integrations. To quantify the reliability, the framework uses the Heaviside step function to eliminate the failure domain and calculates the integration by Monte Carlo Simulation with the function replaced by PCE. The PCE is further combined with Taylor's expansion and the finite difference to compute the reliability sensitivity. Since the design vector may affect the sample set determined by dimension adaptive sparse grid, the update of the sample set is controlled by the norm variations of the design vector. The optimization framework is formed by combining reliability, robustness quantification and sensitivity analysis and the optimization module. The accuracy and efficiency of the reliability quantification, as well as the reliability sensitivity, are verified through a mathematical example, a system of springs, and a cantilever beam. The effectiveness of the framework in solving optimization problems is validated by multiple limit states example, a truss optimization example, an airfoil optimization example and an ONERA M6 wing optimization problem. The results demonstrate that the framework can obtain accurate solutions at the expense of a manageable computational cost.
\end{abstract}

Keywords: polynomial chaos expansion, dimensional adaptive sparse grid, reliability, reliability sensitivity

\section{Introduction}

Due to uncertainties in practice, reliability and robustness have become critical issues in design optimization (Hu et al. 2016; Lopez and Beck 2012; Rackwitz 2001; Verma et al. 2016; Yao et al. 2011). Uncertainties are treated with safety factors or worst-case design methods in a deterministic optimization. However, these methods do not guarantee the required level of safety, and the impact of uncertainty parameters on safety cannot be quantified. Typical uncertainty optimization methods include the reliability-based optimization (RBDO) method and the robust optimization method. The RBDO method assures the safety level by formulating the design feasibility as a reliability constraint ( $\mathrm{Du}$ and Chen 2004). Robust design optimization (RDO) approaches model robustness by correlating the expectation and the variance of the system response with the objective function to make the response insensitive to small changes in system

Corresponding author: Chunlin Gong leonwood@nwpu.edu.cn

1 Shaanxi Aerospace Flight Vehicle Design Key Laboratory, School of Astronautics, Northwestern Polytechnical University, Xi'an 710072, China

2 University of Southampton, Southampton, England, SO17 1BJ, United Kingdom 
parameters (Lelievre et al. 2016).

In uncertainty optimization, quantifications of the reliability and the robustness are needed. For the robustness quantification, commonly used methods are the Taylor-based method, Gaussian quadrature, the stochastic expansion, and simulation-based methods (Beyer and Sendhoff 2007; Keshavarzzadeh et al. 2016; Padulo et al. 2011; Schillings and Schulz 2015; Wu et al. 2018). Primary methods used to determine the reliability metric include the First-Order Reliability Methods (FORM), the Second-Order Reliability Methods (SORM), the probability density evolution method (PDEM), and the simulation-based methods (Li 2016; Park et al. 2015; Yang and Liu 2014). Commonly used uncertainty optimization frameworks are derived from the FORM and the SORM. Based on the coupling between the uncertainty analysis and the optimization process, the methods can be divided into three groups: double loop approach (Keshtegar and Hao 2017; Meng et al. 2015; Youn et al. 2005), single loop approach (Keshtegar and Hao 2016; Keshtegar and Hao 2018) and decoupled approach (Du and Chen 2004; Meng et al. 2016). The framework based on gradient-based optimization has attracted attention in recent years (Keshavarzzadeh et al. 2017; Keshavarzzadeh et al. 2016; Torii et al. 2017).

In gradient-based uncertainty optimization, the gradients of the metric of reliability and robustness are needed. The gradients of robustness are easily derived from the robustness quantification method such as the Taylor-based method of moments, Gaussian quadrature, the stochastic expansion and the simulation-based methods (Beyer and Sendhoff 2007; Keshavarzzadeh et al. 2016; Padulo et al. 2011; Schillings and Schulz 2015; Wu et al. 2018). In the RBDO, the gradient of the reliability with respect to the design variables is also called the reliability sensitivity. Theories and methods of reliability sensitivity analysis are roughly divided into two categories: approximation-based methods and simulation-based methods (Valdebenito et al. 2018; Yanfang et al. 2011; Zhang and Zhang 2016).

Approximation-based methods for reliability sensitivity are mainly based on the FORM or SORM method. Hohenbichler and Rackwitz (1986) developed a method based on the FORM. Enevoldsen and Sørensen (1993) extended the method for parallel systems. However, the method cannot be used to estimate sensitivities of slopes or curvatures, and it is not inapplicable with SORM (Karamchandani and Cornell 1992). Therefore, a method is proposed which is applicable to estimate the reliability sensitivity with the FORM and SORM (Karamchandani and Cornell 1992). Kwak and Lee (1987) presented an analytical approach based on the advanced first-order second-moment (AFOSM) method. Yanfang et al. (2011) proposed a FORM-based approach to solve the problem with strongly non-linear limit state functions with independent normal random variables. To reduce the computational cost, Zhao et al. (2011) employed the support vector regression algorithm to construct the limit state function approximation and used FORM/SORM for the gradient estimation. Moreover, (Papadimitriou and Papadimitriou 2016) proposed a method that combines sparse grid techniques and FORM for uncertainty aerodynamic shape optimization. However, when the problem has high nonlinearity or the random variables do not obey the Gaussian distribution, the FORM and SORM encounter large errors. Moreover, when using FORM and SORM in RBDO, the procedure of searching for the most portable point involves a hard-to-solve nested optimization (Keshavarzzadeh et al. 2016), which makes the method inefficient.

Simulation-based reliability sensitivity methods use samples to directly calculate the reliability metric and combine the FORM or the finite difference to carry out the reliability analysis. Ditlevsen and Bjerager (1989) presented a method using the directional simulation to estimate the reliability metric and the gradient. Wu (1994) proposed an adaptive importance sampling (AIS) method to compute the reliability metric and the gradient. The approach based on a conditional sampling technique was further introduced to improve the efficiency (Au 2005; Ching and Hsieh 2007; Taflanidis and Jia 2011). Jensen et al. (2015) developed a method based on 
subset simulation for high-dimensional problems. Royset and Polak (2004) proposed an algorithm for estimating the gradient using either Monte Carlo simulation (MCS) or Importance Sampling (IS). A procedure was proposed combining the MCS methods and the FORM to ease the computational burden in the estimation of the reliability gradient for the MCS method (Melchers and Ahammed 2004). The method was further extended for the non-normal distribution uncertainty (Ahammed and Melchers 2006). The main disadvantage of the simulation-based approach is that the method usually requires many function evaluations. Simulation-based methods are often used for verification purposes.

The surrogate model has the potential to improve the efficiency and the accuracy, while the polynomial chaos expansion (PCE) has drawn increasing attention in uncertainty optimization (Keshavarzzadeh et al. 2016; Papadimitriou and Papadimitriou 2016; Schillings and Schulz 2015; Torii et al. 2017). PCE is attractive due to its high computational efficiency and the powerful ability to generate functional representations of uncertain variances (Eldred and Burkardt 2009). Du et al. (2014) proposed a method based on non-intrusive PCE and the reliability sensitivity is derived from the FORM/SORM method. This method is suitable for the problem with independent normal distribution uncertainty variables. Wang et al. (2006) used the PCE and MCS for reliability analysis and estimated the reliability sensitivity by integrating the gradient of the distribution function. Torii et al. (2017) proposed algorithms that establish the PCE separately for both reliability analysis and reliability sensitivity analysis. Keshavarzzadeh et al. (2017; 2016) presented a computational framework that uses the Heaviside function as the indicator function, and the sensitivity is computed by the integration of the gradient of the Heaviside function. The derivative of the Heaviside step function is a Dirac delta function, which makes the integration complicated. To calculate the integral, the Heaviside function is approximated by a continuous function, and the parameters are chosen empirically, which leads to a shortage of error analysis of the method.

With the above paragraphs as background, it is evident that PCE is an attractive method for the uncertainty quantification problem. A computational framework based on PCE coupled with gradient optimization becomes attractive for the uncertainty optimization problem. However, there is an opportunity to improve the framework by constructing the PCE more efficiently. Building upon the work (Keshavarzzadeh et al. 2017; Keshavarzzadeh et al. 2016; Torii et al. 2017), the dimensional adaptive sparse grid is introduced to calculate the PCE coefficients in this paper. In addition, in order to eliminate the empirical parameters given by Keshavarzzadeh et al. (Keshavarzzadeh et al. 2017; Keshavarzzadeh et al. 2016) in reliability sensitivity analysis, the proposed framework combines the PCE, the Taylor expansion and the finite difference to evaluate reliability sensitivity, which is derived through analytical derivation. The error criterion of the sensitivity analysis is also established.

The rest of the paper is organized as follows. In Section 2, the formulation of the problem is described, and then the proposed framework is presented in Section 3. Seven test cases are used to validate the effectiveness and the accuracy of the framework in Section 4. Finally, conclusions are given in Section 5.

\section{Problem statement}

Reliability is explicitly considered in the constraints as the success probability of the system or structure, which has to be higher than a given threshold $\mathrm{Pr}_{\text {target }}$. Robustness is considered in the objective function, which is the sum of the expected value $\mathrm{E}$ and the variance Var, of the cost function. The model is defined as follows: 


$$
\begin{aligned}
& \text { Find } \mathbf{d}_{\text {opt }} \text { such that: } \\
& \mathbf{d}_{\text {opt }}=\arg \min _{\mathbf{d}} \mathrm{E}(\operatorname{cost}(\mathbf{d}, \mathbf{x}(\omega)))+\operatorname{Var}(\operatorname{cost}(\mathbf{d}, \mathbf{x}(\omega))) \\
& \text { s.t.: } \operatorname{Pr}_{g, j}\left(\mathrm{~g}_{j}(\mathbf{d}, \mathbf{x}(\omega)) \geq 0\right) \geq \operatorname{Pr}_{\text {target }, j}, j=1,2, \ldots, n g \\
& \quad d_{k}^{\mathrm{L}} \leq d_{k} \leq d_{k}^{\mathrm{U}}, k=1,2, \ldots, n d
\end{aligned}
$$

where $\mathbf{d}$ is the deterministic variable vector, $\mathbf{x}$ is the random variable vector, and $\omega$ is the distribution parameter. The term $\mathbf{d}$ may affect $\mathbf{x}$ when $\mathbf{d}$ is the mean value of $\mathbf{x}$. The term $g>0$ indicates the safe state of the system under investigation, and $\operatorname{Pr}_{g, j}$ is the reliability according to each safe mode. Finally, $n g$ indicates the number of constraint functions, and $n d$ is the dimension of the design vector.

$\operatorname{Pr}_{g}$ is the integral of the probability density function (PDF) $\rho(x)$ over the region $\mathrm{g}(\mathbf{d}, \mathbf{x}(\omega)) \geq 0$. However, the calculation of the integration region is not trivial. Therefore, the Heaviside step function is adopted to eliminate the region of $\mathrm{g}(\mathbf{d}, \mathbf{x}(\omega))<0$. As a result, $\operatorname{Pr}_{g}$ may be defined as Eq.(2).

$$
\operatorname{Pr}_{g}=\int_{\Omega} \mathrm{H}(\mathrm{g}(\mathbf{d}, \mathbf{x})) \rho(\mathbf{x}) d \mathbf{x}
$$

where $\mathrm{H}$ is the Heaviside step function:

$$
\mathrm{H}(g(d, x))=\left\{\begin{array}{l}
1, \mathrm{~g}(\mathbf{d}, \mathbf{x}) \geq 0, \text { safe state } \\
0, \mathrm{~g}(\mathbf{d}, \mathbf{x})<0, \text { failure state }
\end{array}\right.
$$

Since the Heaviside step function is not continuous and the breakpoint $\mathrm{g}(\mathbf{d}, \mathbf{x})=0$ is unknown, Eq. (2) is usually evaluated by MCS. Due to the inefficiency incurred in using MCS directly, an alternative method based on the dimensional adaptive PCE is discussed in the next Section.

\section{A framework based on dimensional adaptive PCE}

\subsection{Non-intrusive PCE}

PCE is widely used in reliability and robustness quantification. (Keshavarzzadeh et al. 2016; Papadimitriou and Papadimitriou 2016; Schillings and Schulz 2015; Torii et al. 2017) PCE is based on multidimensional orthogonal polynomial approximation. For a random vector $\mathbf{x}(\omega)$ with dim random variables and a multi-index $\mathbf{i} \in \mathbb{N}^{\operatorname{dim}}$, the PCE of the function $g(\mathbf{d}, \mathbf{x}(\omega)$ is written as Eq.(4) (Schillings 2010).

$$
g\left(\mathbf{d}, \mathbf{x}(\omega)=\sum_{j=0}^{\infty} \sum_{\mathbf{i} \in\left\{\|i\|_{1}=j\right\}} \tilde{\mathbf{g}}_{\mathbf{i}}(\mathbf{d}) \cdot \mathrm{B}_{\mathbf{i}}(\mathbf{x}(\omega))\right.
$$

where $\|\mathbf{i}\|_{1}=\sum_{j=1}^{\operatorname{dim}} i_{j}$ is the $l_{1}$ norm of the index vector, $\tilde{\mathbf{g}}_{\mathbf{i}}(\mathbf{d})$ is the vector of deterministic coefficients, $\mathrm{B}_{\overrightarrow{\mathbf{i}}}(x(\omega))$ is the polynomial bases determined by the Wiener-Askey scheme (Xiu and Karniadakis 2002), in which the Hermite, Legendre, Laguerre, Jacobi and generalized Laguerre orthogonal polynomials are used to simulate the effects of continuous random variables with normalized, uniform, exponential and gamma probability distributions, respectively. These polynomials are orthogonal with respect to the PDF. For example, the multi-dimensional Hermite bases polynomial is defined as Eq.(5).

$$
\begin{aligned}
\mathrm{B}_{\mathbf{i}}(x) & =\prod_{j=0}^{d i m} \mathrm{H}_{i_{j}}\left(x_{j}\right) \\
& =\prod_{j=0}^{d i m}(-1)^{i_{j}} e^{\frac{x_{j}^{2}}{2}} \frac{d^{n}}{d x_{j}^{n}} e^{\frac{x_{j}{ }^{2}}{2}}
\end{aligned}
$$


The infinite expansion is truncated at a finite number in practice. The $p$-order truncated expansion is written in a term-based form in Eq.(6).

$$
\begin{aligned}
& \mathrm{g}\left(\mathbf{d}, \mathbf{x}(\omega)=\sum_{i=0}^{n_{P C}} \tilde{\mathrm{g}}_{i}(\mathbf{d}) \cdot \Psi_{i}(\mathbf{x}(\omega)),\right. \\
& \Psi_{0}\left(\mathbf{x}(\omega)=\mathrm{B}_{0}(x)=1,\left\{\Psi_{i}(\mathbf{x}(\omega))\right\}_{i=0}^{n_{P C}} \equiv\left\{\mathrm{B}_{\mathbf{i}}(x)\right\}_{\|\|_{1}=0}^{p}\right.
\end{aligned}
$$

As shown in Eq.(6), the PCE separates the solution into the deterministic portion $\tilde{g}_{i}(\mathbf{d})$ and the random portion $\Psi_{i}(\mathbf{x}(\omega)) . n_{P C}$ is the number of expansion terms, that are determined by Eq.(7).

$$
n_{P C}=\frac{(s+p) !}{s ! p !}-1
$$

where $s$ is the number of random variables.

The non-intrusive method that uses the simulation code as a black-box is adopted to calculate the coefficients $\tilde{g}_{i}(\mathbf{d})$. Based on the orthogonal characteristics with respect to $\operatorname{PDF} \rho(\mathbf{x}(\omega))$, the coefficients are evaluated using the projection method as given in Eq.(8).

$$
\tilde{\mathrm{g}}_{i}(d)=\frac{\int_{\Omega} \mathrm{g}\left(\mathbf{d}, \mathbf{x}(\omega) \Psi_{i}(\mathbf{x}(\omega)) \rho(\mathbf{x}(\omega)) d \mathbf{x}\right.}{\int_{\Omega}\left[\Psi_{i}(\mathbf{x}(\omega))\right]^{2} \rho(\mathbf{x}(\omega)) d \mathbf{x}}
$$

After identifying the PCE coefficients, the statistical quantities of interest (QOI) are evaluated by replacing the original function by the PCE. The mean value and variance of the output are obtained from Eq.(9).

$$
\begin{aligned}
& \mathrm{E}(\mathrm{g})=\int_{\Omega} \sum_{i=0}^{n_{p C}} \tilde{\mathrm{g}}_{i}(\mathbf{d}) \cdot \Psi_{i}(\mathbf{x}(\omega)) \rho(\mathbf{x}) d \mathbf{x} \\
& =\tilde{\mathrm{g}}_{i}(\mathbf{d}) \\
& \operatorname{Var}(\mathrm{g})=\mathrm{E}\left((\mathrm{g}-\mathrm{E}(\mathrm{g}))^{2}\right) \\
& =\sum_{i=1}^{n_{p C}}\left(\tilde{\mathrm{g}}_{i}(\mathbf{d})\right)^{2} \cdot \int_{\Omega}\left(\Psi_{i}(\mathbf{x}(\omega))\right)^{2} \rho(\mathbf{x}) d \mathbf{x}
\end{aligned}
$$

The reliability can be quantified through Eq.(10).

$$
\operatorname{Pr}_{\mathrm{g}}=\int_{\Omega} \mathrm{H}\left(\sum_{i=0}^{n_{P C}} \tilde{\mathrm{g}}_{i}(\mathbf{d}) \cdot \Psi_{i}(\mathbf{x}(\omega))\right) \rho(\mathbf{x}) d \mathbf{x}
$$

Using Eq.(8) to obtain the PCE coefficients requires multidimensional integration. Next section will introduce the dimensional adaptive sparse grid to discretize the probabilistic space method and to evaluate the multi-dimensional integral.

\subsection{Dimensional adaptive sparse grid}

The dimensional adaptive sparse grid is one of the quadrature-based approaches for high dimensional integration problems. Quadrature-based approaches generally approximate the integral by weighted summation of function values of the specified interpolation points within the integration domain. For multi-dimensional integral, the most straightforward general technique is to employ a tensor product of one-dimensional quadrature rules, called the full tensor grid method, given in Eq.(11).

$$
\begin{aligned}
Q(k, \operatorname{dim})(\mathrm{g})(\xi) & =\left(Q^{i_{1}} \otimes Q^{i_{2}} \otimes \cdots \otimes Q^{i_{\text {dim }}}\right)(g)(\mathbf{x}), \\
& =\sum_{j_{1}=1}^{m_{i_{1}}} \sum_{j_{2}=1}^{m_{i_{2}}} \cdots \sum_{j_{d i m}=1}^{m_{\text {ditim }}} \mathrm{g}\left(x_{j_{1}}^{i_{1}}, x_{j_{2}}^{i_{2}}, \cdots, x_{j_{d i m}}^{i_{d i m}}\right)\left(w_{j_{1}}^{i_{1}}, w_{j_{2}}^{i_{2}}, \cdots, w_{j_{\text {dim }}}^{i_{\text {dim }}}\right)
\end{aligned}
$$


where $m_{i}$ is the number of interpolation points. $k \in \mathbf{N}_{+}$is the order of the tensor grid. The collection of the multivariate quadrature points can be expressed as a grid given in Eq.(12).

$$
\bigcup_{i_{1}, i_{2}, \cdots, i_{\text {dim }} \leq k} \mathbf{G}^{i_{1}} \times \mathbf{G}^{i_{2}} \times \cdots \times \mathbf{G}^{i_{\text {dim }}}
$$

The full tensor grid method needs $\prod_{j=1}^{d i m} m_{i_{j}}$ function evaluations. It results in the exponential growth of function evaluations as the dimension increases, which is named as the curse of dimensionality (Da Ronch et al. 2011).

The sparse grid method initially developed by Smoljak (1963) aims at overcoming the curse of dimensionality. The sparse grid combines the higher-order integral formula of some dimensions with the lower-order integral formula of other dimensions. With $k \geq \operatorname{dim}$, the sparse grids integrand is given as Eq.(13).

$$
S(k, \operatorname{dim})=\sum_{k-\operatorname{dim}+1 \leq \mid i \|_{1} \leq k}(-1)^{k-\|_{1}} \cdot\left(\begin{array}{l}
\operatorname{dim}-1 \\
k-\|\mathbf{i}\|_{1}
\end{array}\right) \cdot\left(Q^{i_{1}} \otimes Q^{i_{2}} \otimes \cdots \otimes Q^{i_{\text {dim }}}\right)
$$

The collection of the multivariate quadrature points for sparse grids can be expressed as a grid:

$$
\bigcup_{k-\operatorname{dim}+1 \leq|\leq| \|_{1} \leq k} \mathbf{G}^{i_{1}} \times \mathbf{G}^{i_{2}} \times \cdots \times \mathbf{G}^{i_{\text {dim }}} .
$$

In comparison with the full tensor grid method as given in Eq.(12), which combines the univariate quadrature formulas in each dimension up to order $k$, the sparse grid limits the total sum of all dimensions within the range $[k-\operatorname{dim}+1, k]$. For functions from spaces with bounded mixed derivatives up to order $r>1$, the error bound of the full tensor grid method is $O\left(N^{-r}\right)$ and the error bound for the sparse grid method is $O\left(N^{-r} \cdot(\log N)^{(r+1)(d-1)}\right)$ (Schillings 2010). Thus, the sparse grid method is capable of overcoming the curse of dimensionality and is suitable for high dimensional integration problems.

By using the nested rules, a difference formula $\Delta^{i}=Q^{i+1}-Q^{i}$ can be defined with $Q^{0}=0$ and the difference points set $\mathbf{G}_{\Delta}^{i}=\mathbf{G}^{i+1} \backslash \mathbf{G}^{i}$. With the difference formula defined, the sparse grid method can be expressed in an incremental form as Eq.(15)-(16).

$$
\begin{aligned}
S(k, \operatorname{dim}) & =\sum_{\|\|_{1} \leq k} \Delta^{i_{1}} \otimes \Delta^{i_{2}} \otimes \cdots \otimes \Delta^{i_{\text {dim }}} \\
& =S(k-1, \operatorname{dim})+\sum_{\| i_{1}=k} \Delta^{i_{1}} \otimes \Delta^{i_{2}} \otimes \cdots \otimes \Delta^{i_{\text {dim }}}
\end{aligned}
$$

The dimensional adaptive sparse grid based on the incremental form adds points sequentially considering the importance of each dimension (Bungartz and Dirnstorfer 2003; Garcke et al. 2001; Gerstner and Griebel 2003; Klimke 2006). Starting with a coarse sparse grid, the multi-index is collected in the set $\mathbf{I}=\left\{\mathbf{i}: k-\operatorname{dim}+1 \leq\|\mathbf{i}\|_{1} \leq k\right\}$. The sparse grid integral with the index set $\mathbf{I}$ is :

$$
S(k, \operatorname{dim})=\sum_{\mathbf{i} \in \mathbf{I}} \Delta^{i_{1}} \otimes \Delta^{i_{2}} \otimes \cdots \otimes \Delta^{i_{\text {dim }}} .
$$

A forward neighbourhood set of $\mathbf{I}$ is given as

$$
\mathbf{I}_{\text {nei }}=\left\{\mathbf{i}_{\text {nei }}: \mathbf{i}_{\text {nei }}=\mathbf{i}+\mathbf{e}_{j}, \mathbf{i}_{\text {nei }}-\mathbf{e}_{k} \in \mathbf{I}, \mathbf{i} \in \mathbf{I}, 1 \leq j \leq \operatorname{dim}, 1 \leq k \leq \operatorname{dim}\right\} .
$$

After evaluating the PCE coefficients by Eq.(17), statistical QOI are obtained by Eq.(9) and Eq.(10). For a multi-index $\mathbf{i}_{\text {nei }} \in \mathbf{I}_{\text {nei }}$, an error indicator of the QOI can be defined by Eq.(19).

$$
\Delta_{\mathbf{i}_{\text {nei }}}(\mathrm{QOI})=\mathrm{QOI}_{\mathbf{I}+\left\{\mathbf{i}_{\text {nei }}\right\}}-\mathrm{QOI}_{\mathbf{I}}
$$

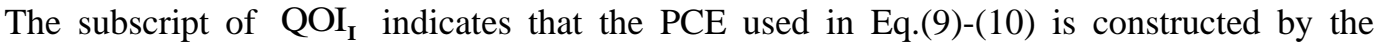
index set $\mathbf{I}$. $\mathbf{i}_{\text {nei }}$ with the maximum quadrature error is sequentially added to $\mathbf{I}$. The 
identification of the multi-index, which causes the maximum quadrature error, can be formulated as an optimization problem given in Eq.(20). The optimization problem is solved by the ergodic method.

$$
\begin{aligned}
& \text { Find } \mathbf{i}_{\text {nei_m }} \text { such that: } \\
& \mathbf{i}_{\text {nei_m }}=\arg \max \Delta_{\mathbf{i}}(\mathrm{QOI}) \\
& \text { s.t.: } \mathbf{i} \in \mathbf{I}_{\text {nei }}
\end{aligned}
$$

In the evaluation of the PCE coefficients, the main advantage of using dimension adaptive refinement strategy is that the PCE can be constructed to be dependent on the problem. This advantage reduces function evaluations.

\subsection{Summary of the Framework}

The process of the quantification of the QOI is summarized in Fig.1. Since this method is based on non-intrusive PCE and dimensional adaptive sparse grids, this method is denoted as NIPCE-DASG. The subscript of $\mathbf{I}_{\mathbf{d}}$ indicates that the design is assigned as $\mathbf{d}$. The initial multi-index set is specified as $\mathbf{I}_{\mathbf{d}}=\left\{\overrightarrow{\mathbf{i}}: k-\operatorname{dim}+1 \leq\|\mathbf{i}\|_{1} \leq k\right\}$, which generates a sparse grid as given in Eq.(14). In this work, $k$ is set to $0 . \varepsilon$ denotes the accuracy required for reliability and robustness quantification problems, which can be assigned according to the accuracy requirements of specific problems.

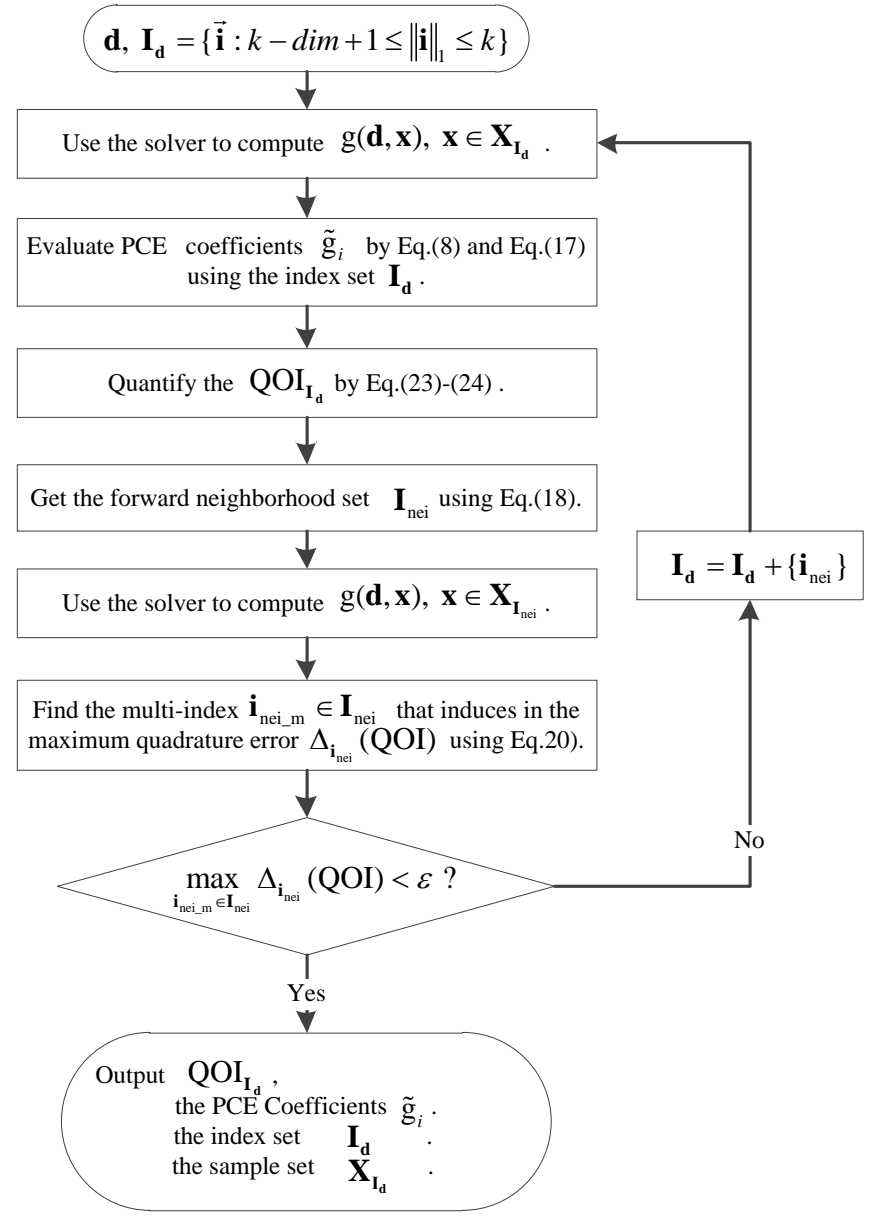

Fig.1 The process of index selection and QOI quantification

The required interpolation samples of the random vector by the dimensional adaptive sparse grid are expressed as a sample set $\mathbf{X}_{\mathrm{I}}=\left\{\mathbf{x}_{1}, \mathbf{x}_{2}, \ldots \mathbf{x}_{n s}\right\}$. The PCE coefficients and the PCE can be expressed as Eq.(21) and Eq.(22), respectively. 


$$
\begin{gathered}
\tilde{\mathrm{g}}_{i}(\mathbf{d})=\sum_{i=0}^{n_{s}} \mathrm{~g}\left(\mathbf{d}, \mathbf{x}_{i}\right) w_{i} \\
\mathrm{~g}\left(\mathbf{d}, \mathbf{x}(\omega)=\sum_{i=0}^{n_{P C}} \sum_{j=0}^{n_{s}} \mathrm{~g}\left(\mathbf{d}, \mathbf{x}_{j}\right) w_{i j} \cdot \Psi_{i}(\mathbf{x}(\omega))\right.
\end{gathered}
$$

Based on the PCE construct by Eq.(21) using the sample set $\mathbf{X}=\left\{\mathbf{x}_{1}, \mathbf{x}_{2}, \ldots \mathbf{x}_{n s}\right\}$, the reliability can be quantified through Eq.(23), and the integral is evaluated through MCS.

$$
\operatorname{Pr}_{g}=\int_{\Omega} \mathrm{H}\left(\sum_{i=0}^{n_{p C}} \sum_{j=0}^{n_{s}} \mathrm{~g}\left(\mathbf{d}, \mathbf{x}_{j}\right) w_{i j} \cdot \Psi_{i}(\mathbf{x}(\omega))\right) \rho(\mathbf{x}) d \mathbf{x}
$$

The mean value and the variance of the output are obtained from Eq.(24).

$$
\begin{aligned}
\mathrm{E}[\mathrm{g}] & =\int_{\Omega}^{n_{p C}} \sum_{i=0}^{n_{s}} \sum_{j=0}^{n_{s}} \mathrm{~g}\left(\mathbf{d}, \mathbf{x}_{j}\right) w_{i j} \cdot \Psi_{i}(\mathbf{x}(\omega)) \rho(\mathbf{x}) d \mathbf{x} \\
& =\sum_{j=0}^{n_{s}} \mathrm{~g}\left(\mathbf{d}, \mathbf{x}_{j}\right) w_{0 j} \\
\operatorname{Var}(\mathrm{g}) & =\mathrm{E}\left[(\mathrm{g}-\mathrm{E}(\mathrm{g}))^{2}\right] \\
& =\sum_{i=1}^{n_{p C}}\left(\sum_{j=0}^{n_{s}} \mathrm{~g}\left(\mathbf{d}, \mathbf{x}_{j}\right) w_{i j}\right)^{2} \cdot \int_{\Omega}\left(\Psi_{i}(\mathbf{x}(\omega))\right)^{2} \rho(\mathbf{x}) d \mathbf{x}
\end{aligned}
$$

To evaluate reliability sensitivity, the gradient of the Heaviside step function is needed. The derivative of the Heaviside step function is the Dirac delta function shown in Eq.(25).

$$
\frac{\partial \mathrm{H}(x)}{\partial x}=\delta(x)= \begin{cases}+\infty, & x=0 \\ 0, & x \neq 0\end{cases}
$$

Therefore, the reliability sensitivity is expressed as:

$$
\frac{\partial \operatorname{Pr}_{g}}{\partial d_{k}}=\int_{\Omega} \delta(\mathrm{g}(\mathbf{d}, \mathbf{x})) \frac{\partial \mathrm{g}(\mathbf{d}, \mathbf{x})}{\partial d_{k}} \rho(\mathbf{x}) d \mathbf{x} .
$$

However, the Dirac delta function makes the computation of the integral complex. The difficulty is to determine the surface of $\mathrm{g}(\mathbf{d}, \mathbf{x})=0$ accurately. Furthermore, MCS is not applicable since it is hard to sample a set randomly with $\mathrm{g}(\mathbf{d}, \mathbf{x})=0$. Therefore, the finite difference given in Eq.(27) is adopted to evaluate the reliability sensitivity.

$$
\frac{\partial \operatorname{Pr}_{g}}{\partial d_{k}} \approx \frac{\operatorname{Pr}_{g}\left(\mathrm{~g}\left(\mathbf{d}+h \mathbf{e}_{k}, \mathbf{x}\right) \geq 0\right)-\operatorname{Pr}_{g}(\mathrm{~g}(\mathbf{d}, \mathbf{x}) \geq 0)}{h}
$$

The Taylor's expansion introduces the function gradient $\partial \mathrm{g}(\mathbf{d}, \mathbf{x}) / \partial d_{k}$ and makes an approximation as Eq.(28).

$$
\mathrm{g}\left(\mathbf{d}+h \mathbf{e}_{k}, \mathbf{x}\right)=\mathrm{g}(\mathbf{d}, \mathbf{x})+\frac{\partial \mathrm{g}(\mathbf{d}, \mathbf{x})}{\partial d_{k}} h
$$

Combining the Heaviside step function, Taylor's expansion and the finite difference method, the reliability sensitivity is expressed as Eq.(29).

$$
\frac{\partial \operatorname{Pr}_{g}}{\partial d_{k}} \approx \frac{\int_{\Omega} \mathrm{H}\left(\mathrm{g}(\mathbf{d}, \mathbf{x})+\frac{\partial \mathrm{g}(\mathbf{d}, \mathbf{x})}{\partial d_{k}} h\right) \rho(\mathbf{x}) d \mathbf{x}-\int_{\Omega} \mathrm{H}(\mathrm{g}(\mathbf{d}, \mathbf{x})) \rho(\mathbf{x}) d \mathbf{x}}{h}
$$

The approximation error for $\mathrm{g}\left(\mathbf{d}+h \mathbf{e}_{k}, \mathbf{x}\right)$ given as Eq.(28) is $O\left(h^{2}\right)$. Using the numerical quadrature method in the reliability sensitivity analysis, the total error of the integration will be the sum of the error caused by the approximation and the integral error caused by the numerical 
quadrature method. The analytical error for Eq.(29) is $O(h)$. Therefore, to achieve the accuracy $O(h)$, the numeric quadrature error should be less than $O\left(h^{2}\right)$.

$\partial \mathrm{g}(\mathbf{d}, \mathbf{x}) / \partial d_{k}$, in Eq.(29), is calculated by Eq.(30), which is the derivative of the PCE given by Eq.(22).

$$
\frac{\partial \mathrm{g}(\mathbf{d}, \mathbf{x})}{\partial d_{k}}=\sum_{i=0}^{n_{p C}} \sum_{j=0}^{n_{s}} \frac{\partial \mathrm{g}\left(\mathbf{d}, \mathbf{x}_{j}\right)}{\partial d_{k}} w_{i j} \cdot \Psi_{i}(\mathbf{x}(\omega))
$$

The gradient of the mean value and the variance of the output are obtained from Eq.(31).

$$
\begin{aligned}
& \frac{\partial \mathrm{E}(\mathrm{g})}{\partial d_{i}}=\sum_{j=0}^{n_{s}} \frac{\partial \mathrm{g}\left(\mathbf{d}, \mathbf{x}_{j}\right)}{\partial d_{i}} w_{0 j} \\
& \frac{\partial \operatorname{Var}(\mathrm{g})}{\partial d_{i}}=\sum_{i=1}^{n_{P C}} 2\left(\sum_{j=0}^{n_{s}} \mathrm{~g}\left(\mathbf{d}, \mathbf{x}_{j}\right) w_{i j}\right) \cdot\left(\sum_{j=0}^{n_{s}} \frac{\partial \mathrm{g}\left(\mathbf{d}, \mathbf{x}_{j}\right)}{\partial d_{i}} w_{i j}\right) \cdot \int_{\Omega}\left(\Psi_{i}(\mathbf{x}(\omega))\right)^{2} \rho(\mathbf{x}) d \mathbf{x}
\end{aligned}
$$

The QOI quantification given in Eq.(23)-(24) and the QOI sensitivity analysis given in Eq.(29)-(31) are coupled with the optimization module to construct an optimization framework. The optimization framework is illustrated in Fig.2.

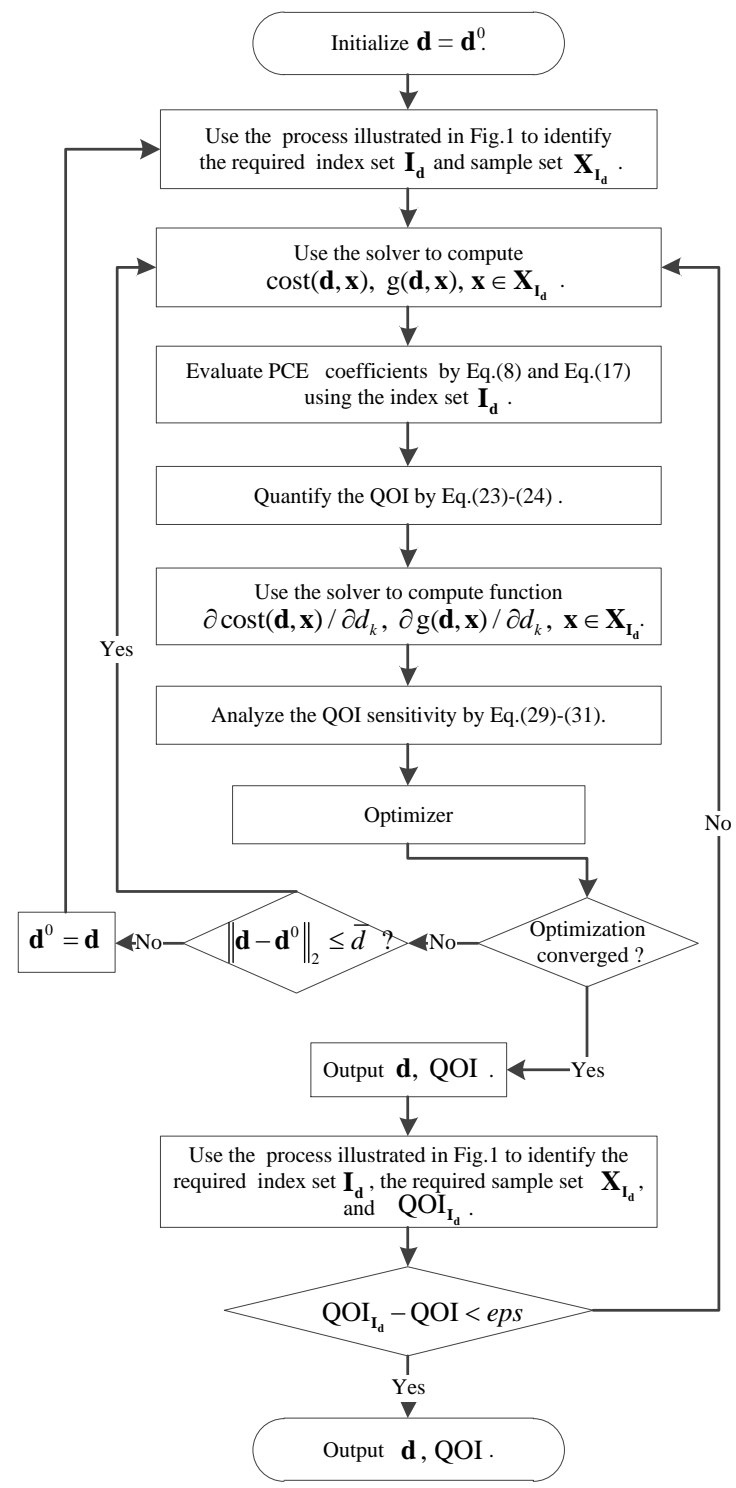

Fig.2 The optimization framework 
In the index selection process given in Fig.1, the samples in set $\mathbf{X}_{\mathbf{I}_{\text {ni }}}$ need to be evaluated, which increases the computational burden. However, the required index set $\mathbf{I}_{\mathbf{d}}$ remains the same when the design varies within a restricted region. Therefore, to reduce the computation induced by the function evaluations of $\mathbf{X}_{\mathrm{I}_{\mathrm{ni}}}$, the update of the index selection process is controlled by the criteria $\left\|\mathbf{d}-\mathbf{d}^{0}\right\|_{2} \leq \bar{d}$, where $\bar{d}$ is the restricted norm variations of the design vector. When the optimization is converged, the QOI is rechecked by the process given in Fig.1. QOI $I_{I_{d}}$ is the output given by the process provided in Fig.1. eps is the acceptable error for QOI. The computation burden caused by the index selection process is released by eliminating the index section process when $\left\|\mathbf{d}-\mathbf{d}^{0}\right\|_{2} \leq \bar{d}$ is satisfied. In the next section, the proposed method is demonstrated by several examples.

\section{Examples and discussion}

\subsection{Mathematical example}

The mathematical example is adopted from Yang and Liu (2014). As is given in Eq.(32)-(33), the two mathematical functions have disjoint failure domains (Yang and Liu 2014). Table 1 and Table 2 give the descriptions of the uncertain parameters. In the reliability quantification problem, FORM, SORM, PDEM and NIPCE-DASG are compared.

$$
\begin{gathered}
\mathrm{g}_{1}\left(x_{1}, x_{2}, x_{3}\right)=0.25\left(\sin \left(x_{1}-3\right)\left(x_{2}-1\right)+\left(x_{3}-1\right)^{2}\right)-1 \\
\mathrm{~g}_{2}\left(x_{1}, x_{2}, x_{3}, x_{4}\right)=0.25\left(\sin \left(x_{1}-3\right)\left(x_{2}-1\right)^{2}+\left(x_{3}-1\right) x_{4}\right)-3
\end{gathered}
$$

Table 1 Parameters of random variables for performance function $g_{1}$

\begin{tabular}{|c|c|c|}
\hline Random variables & Distribution & Ranges \\
\hline$x_{1}$ & uniform & {$[0,10]$} \\
\hline$x_{2}$ & uniform & {$[6,16]$} \\
\hline$x_{3}$ & uniform & {$[0,10]$} \\
\hline
\end{tabular}

Table 2 Parameters of random variables for performance function $\mathrm{g}_{2}$

\begin{tabular}{|c|c|c|}
\hline Random variables & Distribution & Ranges \\
\hline$x_{1}, x_{2}, x_{3}, x_{4}$ & uniform & {$[0,10]$} \\
\hline
\end{tabular}

The reliability quantification problem is defined as follows:

$$
\operatorname{Pr}_{\bar{g}, j}=\iint \mathrm{H}\left(\bar{g}_{j} \geq \mathrm{g}_{j}(\mathbf{x}(\omega))\right) d \mathbf{x}, j=1,2 .
$$

The index selection iteration steps for constructing the PCE are shown in Fig.3. For function $\mathrm{g}_{1}$, the multi-index set is given as $\{(0,0,0),(0,0,1),(1,0,0),(2,0,0),(0,1,0)\}$, and the final error indicator for reliability quantification is $1.429 \times 10^{-3}$. For function $\mathrm{g}_{2}$, the multi-index set is given as $\{(0,0,0,0),(0,1,0,0),(1,0,0,0),(1,1,0,0),(0,0,1,0),(2,0,0,0),(0,0,0,1),(0,0,1,1),(2,1,0,0)\}$, and the final error indicator for reliability quantification is $2.42 \times 10^{-3}$. 


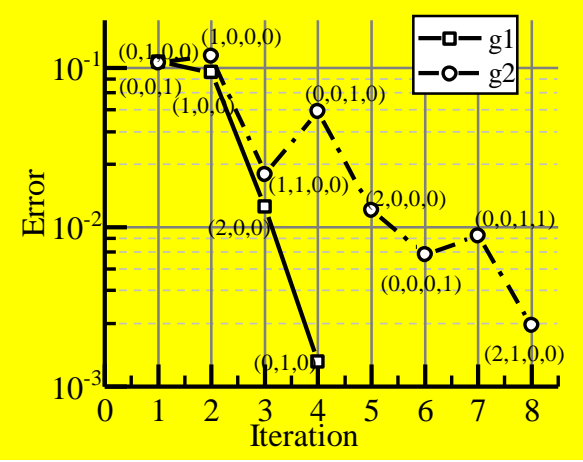

Fig.3 Mathematical example: index selection iteration steps of the functions

The curves of PDF for the mathematical functions using NIPCE-DASG are shown in Fig.4, and these agree well with the ones by MCS.
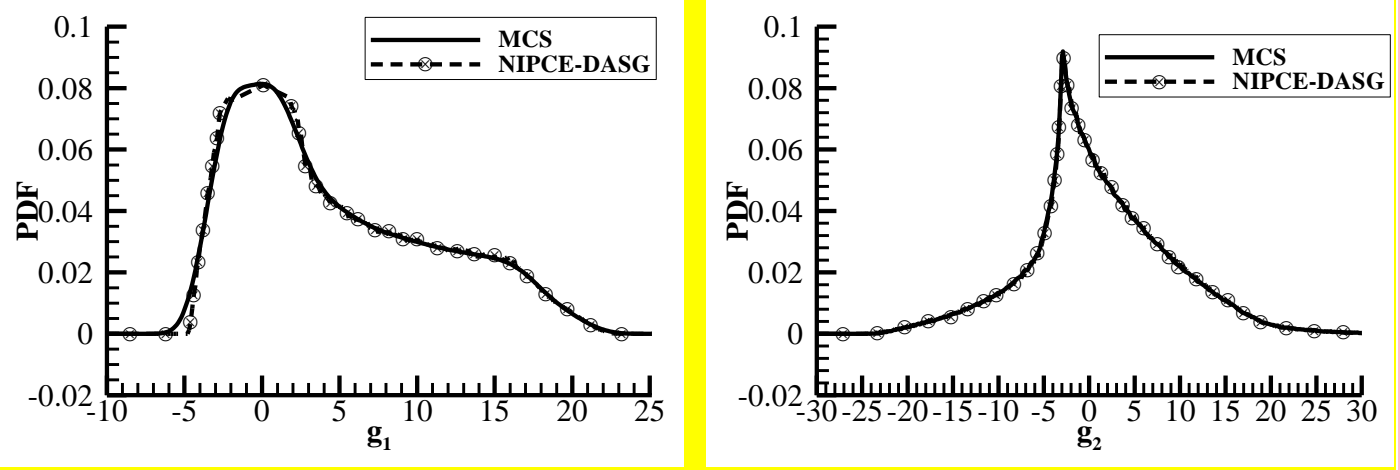

Fig.4 Mathematical example: the PDFs of functions

Table 3 lists the reliability quantification results for different thresholds $\bar{g}$ and compares the results of the different methods.

Table 3 Mathematical example: comparison of methods

\begin{tabular}{|c|c|c|c|c|}
\hline $\bar{g} \geq \mathrm{g}_{1}$ & $\bar{g}=0$ & $\bar{g}=10$ & $\bar{g}=20$ & Number of function calls \\
\hline MCS & 0.2878 & 0.7742 & 0.9929 & $10^{6}$ \\
\hline FORM & 0.3095 & 0.6851 & 0.9560 & 96 \\
\hline SORM & 0.2010 & 0.8619 & 0.9919 & 123 \\
\hline PDEM & 0.2860 & 0.7491 & 0.9901 & 135 \\
\hline NIPCE-DASG & 0.2844 & 0.7740 & 0.9926 & 11 \\
\hline $\bar{g} \geq \mathrm{g}_{2}$ & $\bar{g}=0$ & $\bar{g}=10$ & $\bar{g}=20$ & Number of function calls \\
\hline MCS & 0.4931 & 0.8753 & 0.9875 & $10^{6}$ \\
\hline FORM & 0.3486 & 0.6435 & 0.8739 & 130 \\
\hline SORM & 0.3305 & 0.8818 & 0.9836 & 172 \\
\hline PDEM & 0.4897 & 0.8702 & 0.9905 & 29 \\
\hline NIPCE-DASG & 0.4896 & 0.8745 & 0.9879 & 180 \\
\hline
\end{tabular}

The non-linear inner point (NIP) optimization algorithm in the OPT++ (Meza et al. 2007) library was used to search for the most probable point (MPP) for the FORM and the SORM 
(Eldred et al. 2007). Due to the optimization process in MPP search, FORM and SORM require more function evaluations than the methods using PCE. Also, the first-order (FORM) and second-order approximations (SORM) are less accurate than the PCE. Therefore, the accuracy of the FORM and SORM reliability quantification is lower compared to the method using the PCE. Compared to PDEM, NIPCE-DASG also shows the advantage of obtaining the same level of accuracy with less function evaluation.

\subsection{System of springs}

The spring example was proposed in Keshavarzzadeh et al. (2016). As shown in Fig.5, the system consists of two springs with linear stiffness denoted by $k_{1}$ and $k_{2}$. The system is subject to two external forces $\mathbf{F}=\left(F^{(1)}, F^{(2)}\right)$. The displacement of the two nodes is denoted as $\mathbf{V}=\left(V^{(1)}, V^{(2)}\right)$.

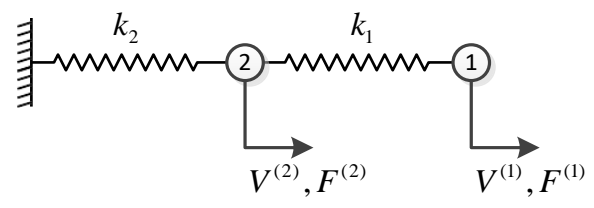

Fig.5 The system of springs problem

In order to show the influence of the uncertainty, two independent random variables $x_{1}$ and $x_{2}$ are included in the expression of spring stiffness and external force as follows:

$$
\begin{aligned}
& k_{1}=\frac{1}{2} d_{1}\left(x_{1}+1\right)+1, \\
& k_{2}=\frac{1}{2} d_{2}\left(x_{1}+1\right)+1, \\
& F^{(1)}=\frac{x_{2}+3}{2}, \\
& F^{(2)}=x_{2}+2
\end{aligned}
$$

where, $\mathbf{d}=\left(d_{1}, d_{2}\right)$ are the deterministic variable that is defined as $\mathbf{d}=(0.5,1.5)$. The strain energy $C=\mathbf{V}^{\mathrm{T}} \mathbf{F}$, widely used in structural optimization, is taken as the performance function. The analytical expressions of $\mathbf{V}$ and $C$ with respect to the random variables are given as follows:

$$
\begin{aligned}
& V^{(1)}=\frac{\left(x_{1}+1\right)\left(x_{2}\left(0.75 d_{1}+0.25 d_{2}\right)+1.75 d_{1}+0.75 d_{2}\right)+2 x_{2}+5}{0.25 d_{1} d_{2}\left(x_{1}+1\right)^{2}+0.5\left(d_{1}+d_{2}\right)\left(x_{1}+1\right)+1} \\
& V^{(2)}=\frac{1.5 x_{2}+3.5}{0.25 d_{2}\left(x_{1}+1\right)+1} \\
& C=\left(\frac{1}{2} x_{2}+\frac{3}{2}\right) V^{(1)}+\left(x_{2}+2\right) V^{(2)}
\end{aligned}
$$

The reliability quantification problem is defined as the probability that $C$ exceeds a specific value $\bar{C}$, as is given in Eq.(37).

$$
\operatorname{Pr}_{C}=\left.\iint \mathrm{H}(C \geq \bar{C})\right|_{d_{1}=0.5, d_{2}=1.5} d x_{1} d x_{2} .
$$

The index selection iteration steps for constructing the PCE when $\bar{C}=20\left(x_{1} \sim \mathrm{U}(-1,1)\right.$ and $\left.x_{2} \sim \mathrm{U}(-1,1)\right)$, are shown in Fig.6. After six iterations, the error indicator as given in Eq.(19) is $7.1 \times 10^{-5}$. The multi-index set is presented as $\{(0,0),(0,1),(1,0),(1,1),(2,0),(2,1)\}$. 

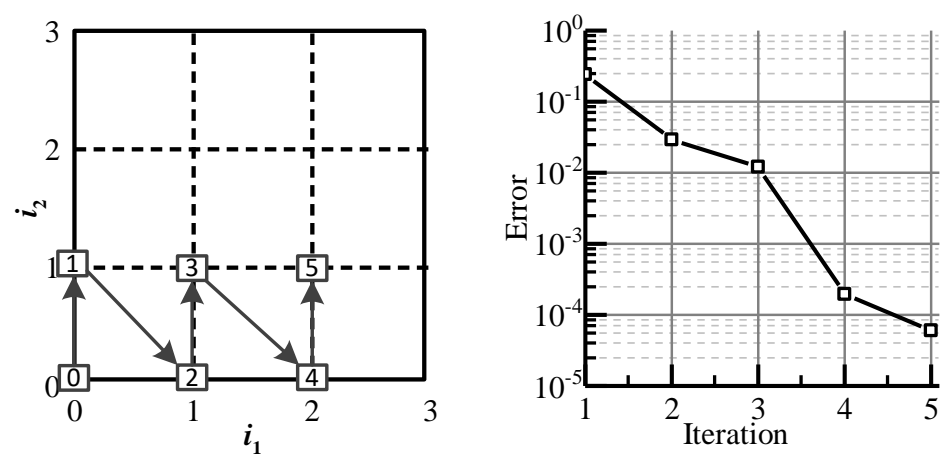

Fig.6 System of springs: index selection iteration steps $\left(x_{1} \sim \mathrm{U}(-1,1), x_{2} \sim \mathrm{U}(-1,1)\right)$

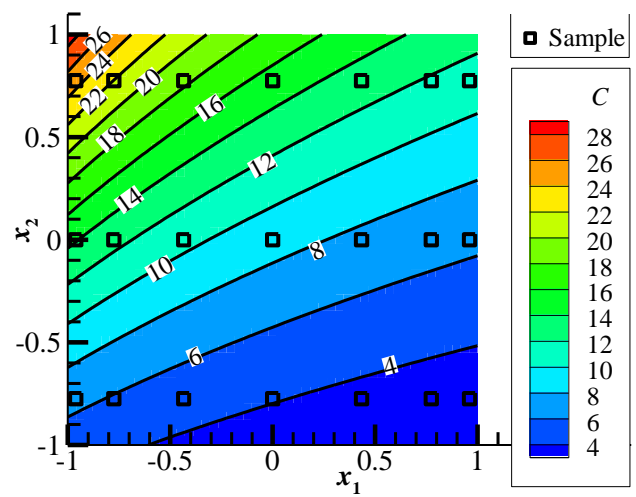

Fig.7 System of springs: samples distribution

Eq.(38) gives the expression of the strain energy when $\mathbf{d}=(0.5,1.5) . x_{1}$ appears mainly in the denominator of the expression, while $x_{2}$ only appears in the numerator, which means that $x_{1}$ induces greater nonlinearity than $x_{2}$. Therefore, as is shown in Fig.7, more values are selected for $x_{1}$ than for $x_{2} .21$ samples are determined through the index set.

$$
C=\frac{2\left(x_{2}+3\right)\left(8 x_{2}+\left(3 x_{2}+8\right)\left(x_{1}+1\right)+20\right)}{16 x_{1}+3\left(x_{1}+1\right)^{2}+32}+\frac{2\left(3 x_{2}+7\right)\left(x_{2}+2\right)}{3 x_{1}+7}
$$

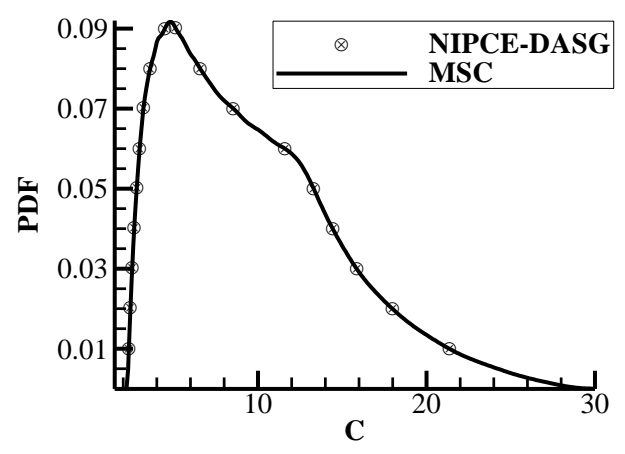

Fig.8 System of springs: PDF of the strain energy $C$

Keshavarzzadeh et al. (2016) studied the non-intrusive and the intrusive PCE with sparse grids. Compared to intrusive methods, the non-intrusive methods allow the solver to be considered as a black-box, which is an essential feature in engineering applications. Hence the non-intrusive method is chosen for comparison. The FORM and the SORM are also selected for comparison. As for the reliability sensitivity analysis, NIPCE-DASH is compared with direct use of MCS in the 
calculation of $\partial \operatorname{Pr}_{C} / \partial d_{1}$.

Table 4 System of springs: comparison of methods

\begin{tabular}{|c|c|c|c|c|c|c|}
\hline $\begin{array}{l}\mathbf{d}=(0.5,1.5) \\
C \geq 20\end{array}$ & Method & $\begin{array}{l}\text { Number of } \\
\text { evaluations } \\
\text { for } \operatorname{Pr}_{C}\end{array}$ & $\operatorname{Pr}_{C}$ & $\begin{array}{l}\text { Relative Error } \\
\text { of } \operatorname{Pr}_{C}\end{array}$ & $\partial \operatorname{Pr}_{C} / \partial d_{1}$ & $\begin{array}{c}\text { Relative } \\
\text { Error of } \\
\partial \operatorname{Pr}_{C} / \partial d_{1}\end{array}$ \\
\hline \multirow{6}{*}{$\begin{array}{l}x_{1} \sim \mathrm{U}(-1,1) \\
x_{2} \sim \mathrm{U}(-1,1)\end{array}$} & Analytical & -— & 0.0472 & -— & -0.00683742 & - - \\
\hline & MCS & $10^{6}$ & 0.047208 & $0.0169 \%$ & -0.00642 & $6.1049 \%$ \\
\hline & FORM & 40 & 0.07310775 & $54.8893 \%$ & - & -— \\
\hline & SORM & 48 & 0.048613548 & $2.9948 \%$ & -— & -— \\
\hline & $\begin{array}{l}\text { Non-intrusive } \\
\text { method in } \\
\text { Keshavarzzadeh } \\
\text { et al. (2016) }\end{array}$ & 33 & 0.0471 & $0.2119 \%$ & -— & -— \\
\hline & NIPCE-DASG & 21 & 0.047205 & $0.0106 \%$ & -0.0064 & $6.3974 \%$ \\
\hline \multirow{4}{*}{$\begin{array}{l}x_{1} \sim \mathrm{U}(1,1) \\
x_{2} \sim \mathrm{N}(0,1)\end{array}$} & MCS & $10^{6}$ & 0.1327 & $\bar{\square}$ & -0.0181 & $\bar{\square}$ \\
\hline & FORM & 30 & 0.140902 & $6.18 \%$ & $\bar{\square}$ & $\bar{\square}$ \\
\hline & SORM & 38 & 0.129039 & $2.76 \%$ & $\bar{\square}$ & $\bar{\square}$ \\
\hline & NIPCE-DASG & 13 & 0.132187 & $0.39 \%$ & -0.0189 & $4.42 \%$ \\
\hline
\end{tabular}

It can be seen from the results in Table 4 that the accuracy of FORM and SORM reliability quantification is lower than that of PCE. Compared to the non-intrusive method of Keshavarzzadeh et al. (2016), DASG's goal-oriented features lead to fewer calculations required by NIPCE-DASG. As for the reliability sensitivity analysis, the accuracy of the proposed method is at the same level of MCS.

\subsection{Cantilever Beam}

The cantilever beam problem shown in Fig.9 is referenced from Yi et al. (2008). The beam has a length of $L$ and the dimensions of the cross section define the design vector $\mathbf{d}=(b, h)$. The cantilever beam is subject to two external forces $\mathbf{F}=\left(F^{(1)}, F^{(2)}\right)$. The uncertainty is induced by the external forces, the elastic modulus $E$, and the yield strength $y$.

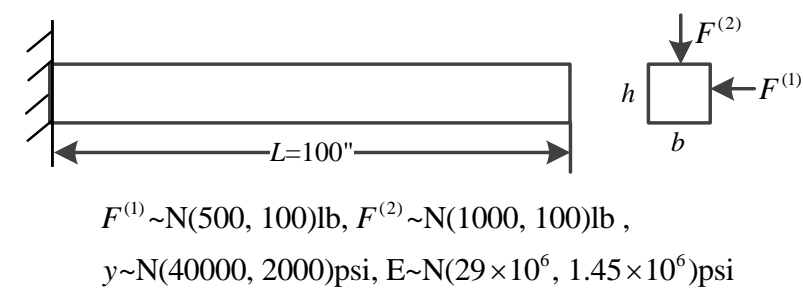

Fig. 9 The cantilever beam optimization problem

$$
\begin{aligned}
& \mathrm{g}_{1}(\mathbf{d}, \mathbf{x})=y-\left(\frac{600}{b h^{2}} F^{(2)}+\frac{600}{b^{2} h} F^{(1)}\right) \\
& \mathrm{g}_{2}(\mathbf{d}, \mathbf{x})=D_{0}-\frac{4 L^{3}}{E b h} \sqrt{\left(\frac{F^{(2)}}{h^{2}}\right)^{2}+\left(\frac{F^{(1)}}{b^{2}}\right)^{2}}
\end{aligned}
$$

As is given in Eq.(39), there are two functions concerned. $\mathrm{g}_{1}(\mathbf{d}, \mathbf{x})$ is the constraint on the maximum stress which is at the fixed end of a corner of the cantilever, where $y$ is the allowable stress. $\mathrm{g}_{2}(\mathbf{d}, \mathbf{x})$ is the tip displacement, where $D_{0}=2.5$ in is the permissible displacement. The design vector is defined as $\mathbf{d}=[2.0,4.0]$. 


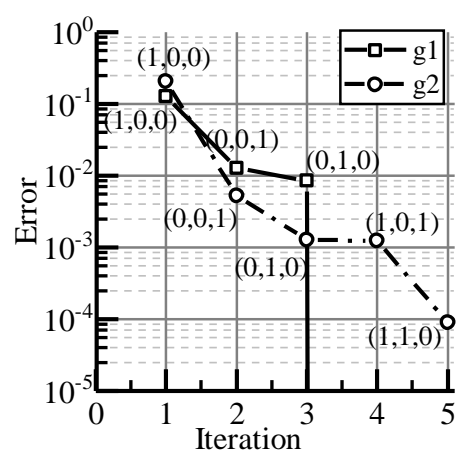

Fig.10 Cantilever beam: index selection iteration steps of the functions

The index selection for the PCE construction to quantify the reliability is shown in Fig.10. The error is $\Delta_{i}(\mathrm{QOI})$ defined by Eq.(19). The fourth index of the $\mathrm{g}_{1}$ is $(0,0,2)$, while the error is zero. Since $g_{1}$ is linear with respect to the random variables, $g_{1}$ is also normally distributed and the PDF can be easily obtained. Seven samples identified by the dimension adaptive grid are capable of making the PCE an accurate agent. Therefore, seven samples are used to construct the PCE. However, $\mathrm{g}_{2}$ is non-linear with respect to the random variables, there are more index selection steps for $\mathrm{g}_{2}$ than $\mathrm{g}_{1}$. It is apparent that the PCE with dimensional adaptive grid method can identify the non-linearity. As given in Fig.11, the proposed method coincides with analytical analysis and MCS in evaluating the PDFs of $g_{1}$ and $g_{2}$.
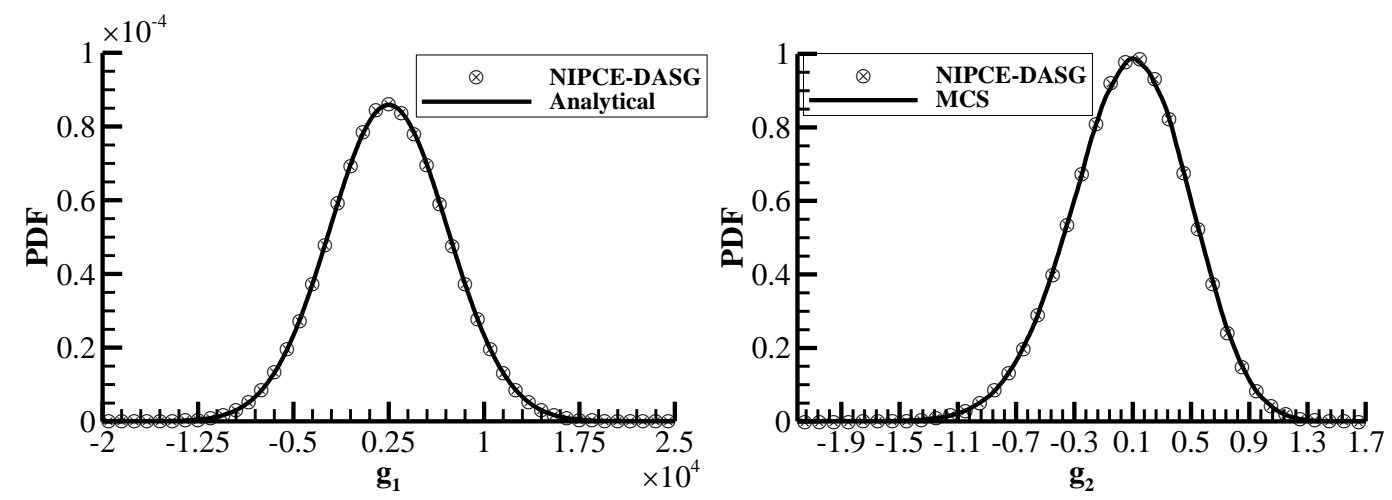

Fig.11 Cantilever beam: the PDF of the functions

Table 5 Cantilever beam: accuracy verifications on reliability quantification and sensitivity analysis

\begin{tabular}{|l|c|c|c|c|c|c|}
\hline$d=[2.0,4.0]$ & \multicolumn{3}{|c|}{$\mathrm{g}_{1}(\mathbf{d}, \mathbf{x}) \geq 0$} & \multicolumn{3}{c|}{$\mathrm{g}_{2}(\mathbf{d}, \mathbf{x}) \geq 0$} \\
\hline Method & MCS & NIPCE-DASG & Error & MCS & NIPCE-DASG & Error \\
\hline Number of evaluations for $\operatorname{Pr}_{C}$ & $10^{6}$ & 7 & -- & $10^{6}$ & 15 & -- \\
\hline $\operatorname{Pr}_{C}$ & 0.7048 & 0.7049 & -0.0001 & 0.5840 & 0.5843 & -0.0005 \\
\hline$\partial \operatorname{Pr}_{C} / \partial d_{1}$ & 2.19 & 2.2280 & -0.0380 & 3.09 & 3.1291 & -0.0127 \\
\hline$\partial \operatorname{Pr}_{C} / \partial d_{2}$ & 1.08 & 1.0740 & 0.0060 & 0.8287 & 0.8301 & -0.0017 \\
\hline
\end{tabular}

In Table 5, the results of the reliability quantification and sensitivity analysis evaluated by NIPCE-DASG and MCS are compared. The maximum reliability error is at the level of $10^{-4}$ and the maximum sensitivity error is at the level of $10^{-2}$. Only seven samples are needed for the linear function $\mathrm{g}_{1}(\mathbf{d}, \mathbf{x})$, and 21 samples are required for the non-linear function $\mathrm{g}_{2}(\mathbf{d}, \mathbf{x})$. The results indicate that the NIPCE-DASG achieves good efficiency and provides excellent accuracy in 
reliability quantification and sensitivity analysis.

The optimization problem defined in Eq.(40) is to minimize the volume while satisfying probabilistic constraints on the maximum stress and the tip displacement.

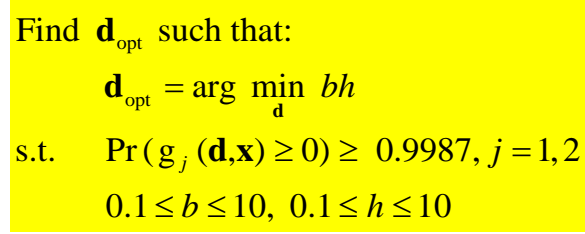

The optimization results by the different method are listed in Table 6 . The results by PMA based methods and RIA based methods are adopted from Yi et al. (2008). The computational cost of each approach is measured by the number of constraint function evaluations (NCE) required to obtain the optimum design.

Table 6 Cantilever beam: uncertainty optimization methods comparison

\begin{tabular}{|c|c|c|c|c|c|c|}
\hline $\begin{array}{c}\text { Initial } \\
\text { solution }\end{array}$ & & PMA bi-level & PMA with SAP & RIA bi-level & RIA with SAP & Proposed method \\
\hline \multirow{2}{*}{$\begin{array}{c}\mathrm{d}(0)= \\
(2.0,4.0)\end{array}$} & NCE & 520 & 176 & 328 & 128 & 176 \\
\hline & $\begin{array}{l}\text { Optimal } \\
\text { solution }\end{array}$ & $\begin{array}{c}9.528 \\
(2.450,3.889) \\
\end{array}$ & $\begin{array}{c}9.527 \\
(2.453,3.884) \\
\end{array}$ & $\begin{array}{c}9.528 \\
(2.439,3.906) \\
\end{array}$ & $\begin{array}{c}9.529 \\
(2.439,3.907) \\
\end{array}$ & $\begin{array}{c}9.5326 \\
(2.3347,4.0830) \\
\end{array}$ \\
\hline \multirow{2}{*}{$\begin{array}{c}\mathrm{d}(0)= \\
(3.0,3.0)\end{array}$} & $\mathrm{NCE}$ & 448 & 224 & 1240 & 432 & 286 \\
\hline & $\begin{array}{l}\text { Optimal } \\
\text { solution }\end{array}$ & $\begin{array}{c}9.528 \\
(2.453,3.884) \\
\end{array}$ & $\begin{array}{c}9.527 \\
(2.455,3.880) \\
\end{array}$ & $\begin{array}{c}9.528 \\
(2.441,3.903) \\
\end{array}$ & $\begin{array}{c}9.532 \\
(2.437,3.911) \\
\end{array}$ & $\begin{array}{c}9.5330 \\
(2.4516,3.8885) \\
\end{array}$ \\
\hline \multirow{2}{*}{$\begin{array}{c}\mathrm{d}(0)= \\
(5.0,5.0)\end{array}$} & $\mathrm{NCE}$ & 584 & 336 & 1096 & 704 & 374 \\
\hline & $\begin{array}{l}\text { Optimal } \\
\text { solution }\end{array}$ & $\begin{array}{c}9.529 \\
(2.436,3.912) \\
\end{array}$ & $\begin{array}{c}9.529 \\
(2.438,3.908) \\
\end{array}$ & $\begin{array}{c}9.523 \\
(2.451,3.885) \\
\end{array}$ & $\begin{array}{c}9.528 \\
(2.452,3.887) \\
\end{array}$ & $\begin{array}{c}9.5237 \\
(2.4840,3.8340)\end{array}$ \\
\hline
\end{tabular}

As shown in Table 6, the proposed framework and the PMA with SAP have similar efficiency. In the optimization process, $\partial \mathrm{g}(\mathbf{d}, \mathbf{x}) / \partial d_{k}$ is obtained by the finite difference method which has low efficiency. More efficient design sensitivity analysis can improve the efficiency of the proposed framwork. The initial solution has a small impact on the final solution.

\subsection{Multiple limit states optimization}

The multiple limit states optimization problem given as Eq.(41) is referenced from Torii et al. (2017). The problem is performed to investigate the effectiveness of the proposed approach in handling multiple nonlinear limit state functions. The efficiency of the proposed approach is assessed against available methods in the literature.

$$
\begin{aligned}
& \text { Find } \mathbf{d}_{\text {opt }} \text { such that: } \\
& \quad \begin{array}{l}
\mathbf{d}_{\text {opt }}=\arg \min _{\mathbf{d}} d_{1}+d_{2} \\
\text { s.t. } \quad \operatorname{Pr}\left(0 \geq \mathrm{g}_{j}(\mathbf{d}, \mathbf{x})\right) \leq 0.0228, j=1,2,3 \\
\\
0.0 \leq d_{1} \leq 10,0.0 \leq d_{2} \leq 10
\end{array} \\
& \text { where } \\
& \qquad \begin{array}{l}
\mathrm{g}_{1}=x_{1}^{2} x_{2} / 20.0-1.0 \\
\mathrm{~g}_{2}=\left(x_{1}+x_{2}-5\right)^{2} / 30.0+\left(x_{1}-x_{2}-12\right)^{2} / 120.0-1 \\
\mathrm{~g}_{3}=80.0 /\left(x_{1}^{2}+8 x_{2}+5\right)-1 \\
x_{i} \sim \mathrm{U}\left(d_{i}-0.6 \sqrt{3}, d_{i}+0.6 \sqrt{3}\right), i=1,2
\end{array}
\end{aligned}
$$

Results obtained by the proposed approach and referenced from Torii et al. (2017) are listed in Table 7. The maximum probability of failure among the three limit states is presented. The NCE measures the computational cost of each approach. 
Table 7 Multiple limit states optimization: comparison of uncertainty optimization methods

\begin{tabular}{|c|c|c|c|c|}
\hline \multicolumn{2}{|c|}{ Approaches } & Optimal solution & NCE & P(MCS) \\
\hline \multirow{2}{*}{ FORM based } & PMA & $7.172(3.626,3.545)$ & 604 & 0.0127 \\
\cline { 2 - 5 } & PMA-SAP & $6.869(3.521,3.348)$ & 132 & 0.0498 \\
\hline \multirow{2}{*}{ Polynomial } & First order & $7.194(3.627,3.567)$ & 636 & 0.0114 \\
\cline { 2 - 5 } & Second order & $7.061(3.581,3.479)$ & 1485 & 0.0229 \\
\hline \multicolumn{2}{|r|}{ Proposed framework } & $7.0645(3.5825,3.4820)$ & 304 & 0.0227 \\
\hline
\end{tabular}

In this case, it can be noticed that the FORM-based optimization approaches are inaccurate. The optimal solution by the PMA is too conservative while the PMA-SAP does not meet the reliability constraints. Compared to the polynomial based method, the DASG used in the proposed framework which has goal-oriented features leads to fewer functions calculations. The proposed framework is very competitive in efficiency and accuracy in solving this problem.

\subsection{Truss optimization}

The truss optimization problem in Fig.12 is referenced from Duan (2006). The system consists of 7 bars and is subject to two external forces $\mathbf{F}=\left(F^{(1)}, F^{(2)}\right)$. Assume that bars 1, 2 have the same cross-sectional area $A_{1}$, bars 3, 4, 5 have the same cross-sectional area $A_{2}$, and bars 6 , 7 have the same cross-sectional area $A_{3}$. Uncertainties in the system are quantified by the independent normal random variables $\mathrm{N}(0,1)$ as $\mathbf{x}=\left(x_{1}, x_{2}, x_{3}, x_{4}, x_{5}, x_{6}, x_{7}, x_{8}\right)$. The random variables are collected as $x_{E}=x_{1}, x_{D}=\left(x_{2}, x_{3}, x_{4}\right), x_{G}=\left(x_{5}, x_{6}\right)$ and $x_{L}=\left(x_{7}, x_{8}\right)$, which correspond to the uncertainties in the modulus of elasticity $E_{y}$, the actual cross-sectional areas $A$, the node coordinates $N_{\text {xy }}$, and the external nodes $\mathbf{F}$. The design vector is $\mathbf{d}=\left(d_{1}, d_{2}, d_{3}\right)$, where the components are the mean values of the cross-sectional areas.

$$
\begin{aligned}
& E_{\mathrm{y}}\left(x_{\mathrm{E}}\right) /\left(\mathrm{kN} / \mathrm{cm}^{2}\right)=20000\left(1+0.2 x_{1}\right) \\
& A\left(x_{D}\right) /\left(\mathrm{cm}^{2}\right)=\left(A_{1}\left(x_{2}\right), A_{2}\left(x_{3}\right), A_{3}\left(x_{4}\right)\right)=\left(d_{1}+0.03 x_{2}, d_{2}+0.03 x_{3}, d_{3}+0.03 x_{4}\right) \\
& N_{\mathrm{xy}}\left(x_{G}\right) /(\mathrm{m})=\left[\begin{array}{cc}
0 & 0 \\
3+0.0827 x_{5} & 0.0963 x_{6} \\
6+0.0699 x_{5} & 0 \\
3+0.0742 x_{5} & 3+0.0684 x_{6} \\
6 & 3+0.0985 x_{6}
\end{array}\right] \\
& F\left(x_{\mathrm{L}}\right) /(\mathrm{kN})=\left(F^{(1)}\left(x_{\mathrm{L}}\right), F^{(2)}\left(x_{\mathrm{L}}\right)\right)=\left(12.5+12.5 x_{7}, 25+25 x_{8}\right)
\end{aligned}
$$

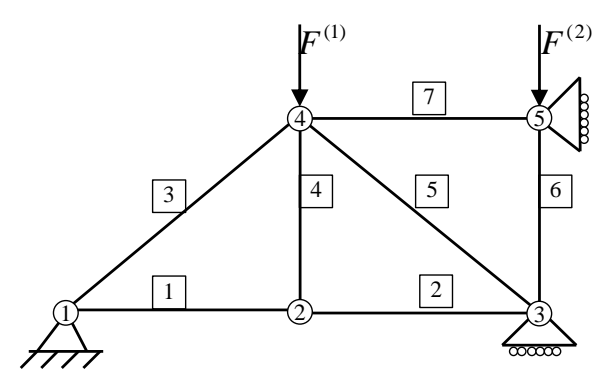

Fig.12 The truss optimization problem

The optimization problem is to minimize the volume ( $\mathrm{Vol}$ ) while satisfying probabilistic constraints on the maximum allowable strain energy. The objective function is the sum of the Vol mean value of $\mathrm{Vol} \mathrm{E}(\mathrm{Vol})$, and its variance $\mathrm{Var}(\mathrm{Vol})$. By adding the variance to the objective, the significant performance fluctuations relative to the random variables can be avoided. The optimization problem is formulated by Eq.(43). 
Find $\mathbf{d}_{\mathrm{opt}}$ such that:

$$
\begin{array}{ll} 
& \mathbf{d}_{\text {opt }}=\arg \min _{\mathbf{d}} \mathrm{E}(\operatorname{Vol}(\mathbf{d}, \mathbf{x}))+0.01 * \operatorname{Var}(\operatorname{Vol}(\mathbf{d}, \mathbf{x})) \\
\text { s.t. } \quad & \operatorname{Pr}(\mathrm{g}(\mathbf{d}, \mathbf{x}) \geq 0) \geq 0.9, \\
& (0.1,0.1,0.1) \leq \mathbf{d} \leq(20,20,20) \\
\text { where } & \mathrm{g}(\mathbf{d}, \mathbf{x})=25-C(\mathbf{d}, \mathbf{x})
\end{array}
$$

The initial design vector is defined as $\mathbf{d}^{(0)}=(5.0,10.0,15.0)$.

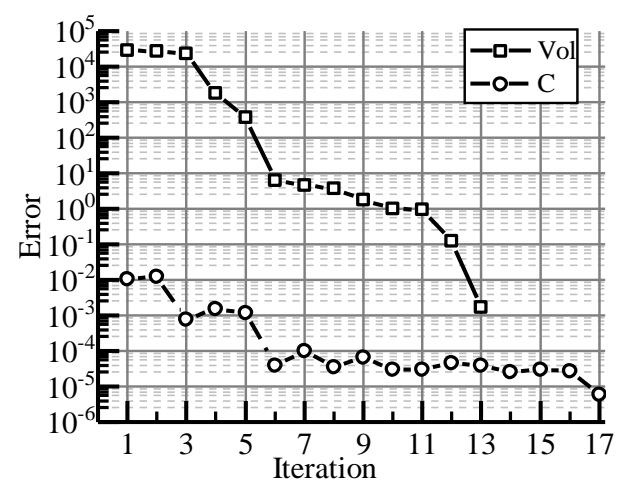

Fig.13 Truss: index selection iteration steps

The index selection iteration steps for constructing the PCE of the truss problem are shown in Fig.13. During the iterations, the QOI for $\mathrm{Vol}$ is the $\operatorname{Var}(\mathrm{Vol})$, and the QOI for the strain energy is $\operatorname{Pr}(C<25)$. For Vol, after 13 steps the error falls from $2.8 \times 10^{4}$ to $1.6 \times 10^{-3}$, while $\operatorname{Var}(\operatorname{Vol})$ is $1.6 \times 10^{5}$. The final relative error for $\operatorname{Var}(\mathrm{Vol})$ is $1 \times 10^{-8}$, which is acceptable. The number of samples to generate the PCE of $\mathrm{Vol}$ is 44 . For the strain energy, the final error for $\operatorname{Pr}(C<25)$ is $6 \times 10^{-6}$. The sample number to construct the PCE of $\mathrm{C}$ is 52 .

Table 8 Truss: QOI comparison of the initial and optimal solutions

\begin{tabular}{|c|c|c|c|c|c|c|}
\hline & $d_{1}$ & $d_{2}$ & $d_{3}$ & $\mathrm{E}(\mathrm{Vol}) / \mathrm{cm}^{3}$ & $\operatorname{Var}(\operatorname{Vol}) / \mathrm{cm}^{6}$ & $\operatorname{Pr}(\mathrm{g}(\mathbf{d}, \mathbf{x}) \geq 0)$ \\
\hline Initial & 5 & 10 & 15 & 23489.2 & 161619 & 0.9420 \\
\hline Uncertainty optimization & 3.4040 & 7.8270 & 13.4058 & 19078.4 & 149950.9 & 0.9042 \\
\hline Deterministic optimization & 0.7186 & 1.4787 & 3.1726 & 4033.71 & 115922 & 0.6580 \\
\hline
\end{tabular}

Fig.14 shows that the optimization converges in 8 iterations. The optimal solution is $d=(3.4040,7.8270,13.4058)$. The initial and optimal solutions are compared in Table 8 . It is found that $\mathrm{E}(\mathrm{Vol})$ and $\operatorname{Var}(\mathrm{Vol})$ decrease while the $\operatorname{Pr}(\mathrm{g}(\mathbf{d}, \mathbf{x}) \geq 0)$ constraint is satisfied for the uncertainty optimization. Compared with the deterministic optimal solution, the optimal solution of the uncertainty optimization has a larger volume, which is necessary for satisfying the reliability constraint.
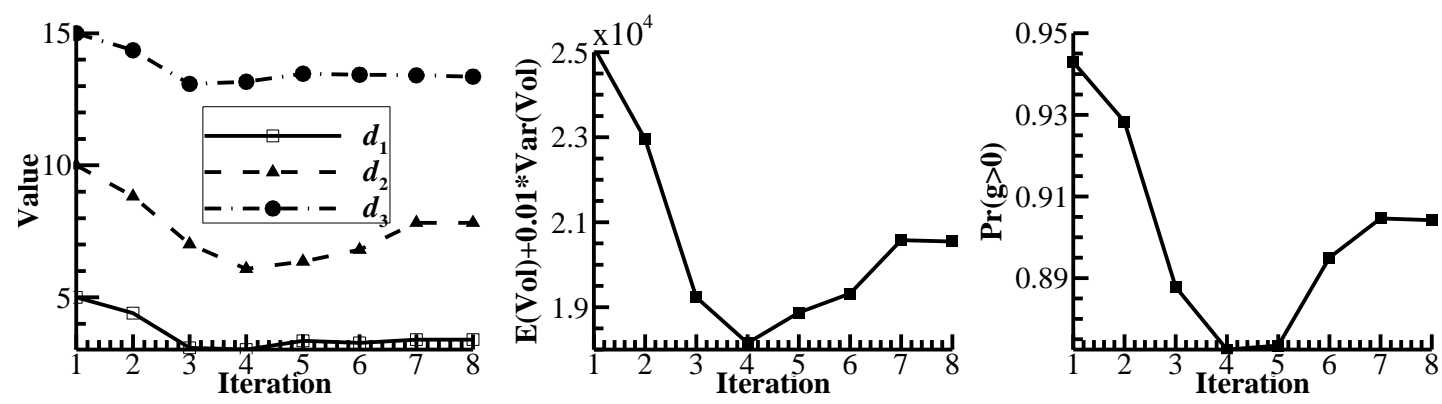

Fig.14 Truss: iteration for solving the uncertainty optimization problem 

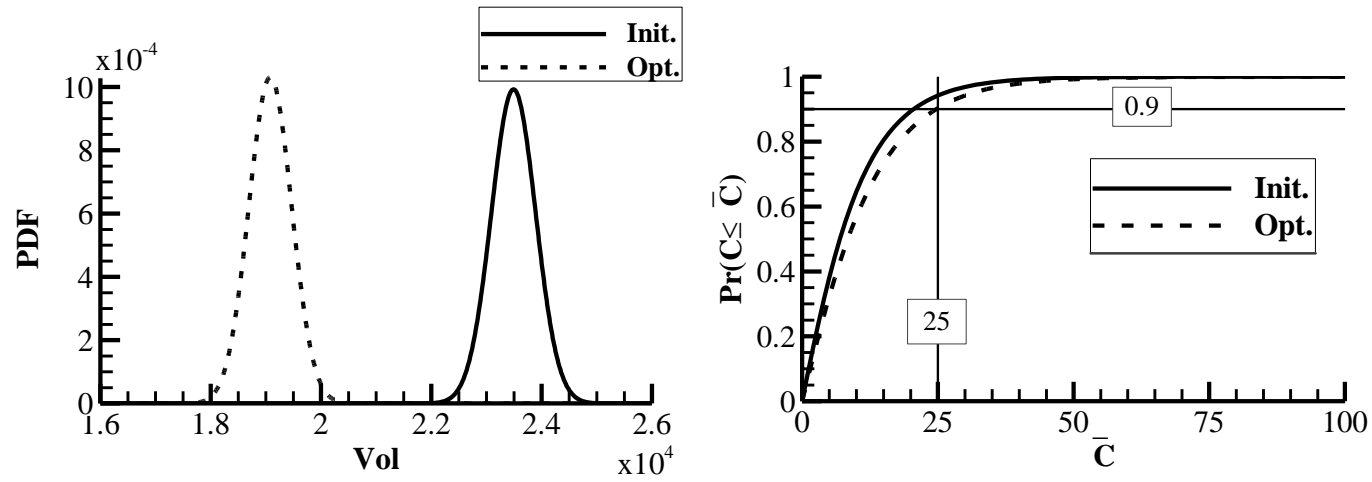

Fig.15 Truss: MCS results of the initial (Init.) and uncertainty optimal (Opt.) solutions

To further verify the uncertainty optimization, the MCS results of the initial and optimal solutions are compared in Fig.15. For the strain energy, the cumulative function over the range $[0,50]$ is clearly decreased. The MCS results of $\operatorname{Pr}(\operatorname{g}(\mathbf{d}, \mathbf{x}) \geq 0)$ is 0.9035 . For the volume, the mean is $19,078.4 \mathrm{~cm}^{3}$, and the variance is $149,803 \mathrm{~cm}^{6}$. Both the accuracies of the constraint and the objective function are acceptable.

\subsection{Airfoil optimization}

In flight, changes in freestream conditions, such as velocity, the angle of attack (AoA), pressure, etc., may occur randomly. Therefore, in aerodynamic optimization, the uncertainty should be considered. The shape optimization of a RAE2822 profile in transonic, inviscid flow is investigated in the following example.

Table 9 Airfoil optimization: freestream conditions

\begin{tabular}{|c|c|c|c|}
\hline & Distribution & Mean & Variance \\
\hline Velocity/(m/s) & Normal & 248.08 & 10 \\
\hline $\mathrm{AoA} /(\mathrm{o})$ & Normal & 2 & 0.5 \\
\hline Pressure $/(\mathrm{Pa})$ & Normal & 101325.0 & 5066.25 \\
\hline
\end{tabular}

The geometry is parameterized using 18 Hicks-Henne bump functions. The $\mathrm{x}$-axis coordinates of the control points are fixed while the y-axis coordinates are the design variables. The details are shown in Table 10. The sketching of geometric parameters is shown in Fig.16, in which the solid black points represent the control points of the bumps. The movements along the $y$-axis of the control points are defined as design variables within a given design range of $[-0.01$, $0.01]$.

Table 10 Airfoil optimization: specification of the design variables

\begin{tabular}{|c|c|c|c|c|c|c|c|c|c|}
\hline Des. Var. (Index) & 1 & 2 & 3 & 4 & 5 & 6 & 7 & 8 & 9 \\
\hline X-axis $(\boldsymbol{x} / \boldsymbol{C})$ & 0.05 & 0.1 & 0.2 & 0.3 & 0.4 & 0.5 & 0.6 & 0.7 & 0.8 \\
\hline Des. Var. (Index) & 10 & 11 & 12 & 13 & 14 & 15 & 16 & 17 & 18 \\
\hline X-axis $(\boldsymbol{x} / \boldsymbol{C})$ & 0.05 & 0.1 & 0.2 & 0.3 & 0.4 & 0.5 & 0.6 & 0.7 & 0.8 \\
\hline
\end{tabular}

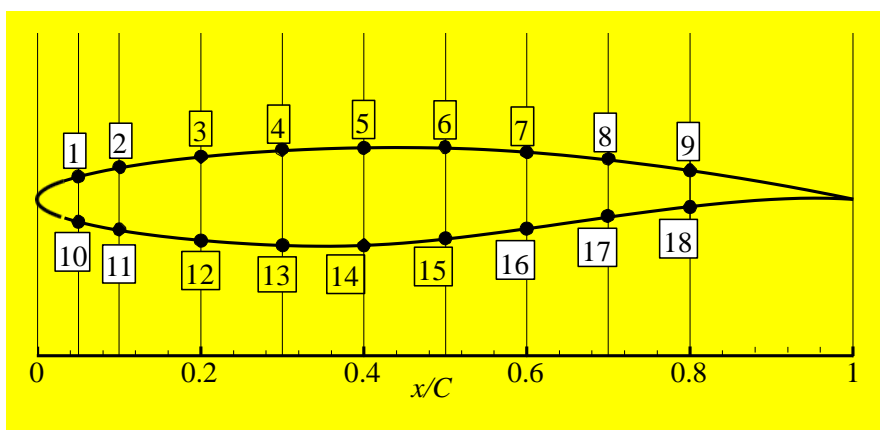

Fig.16 Parameterization of the RAE2822 airfoil 
The optimization problem is to minimize the drag while satisfying probabilistic constraints on the lift. The objective function is the sum of the drag mean value $\mathrm{E}(\mathrm{Drag})$ and its variance $\operatorname{Var}($ Drag).

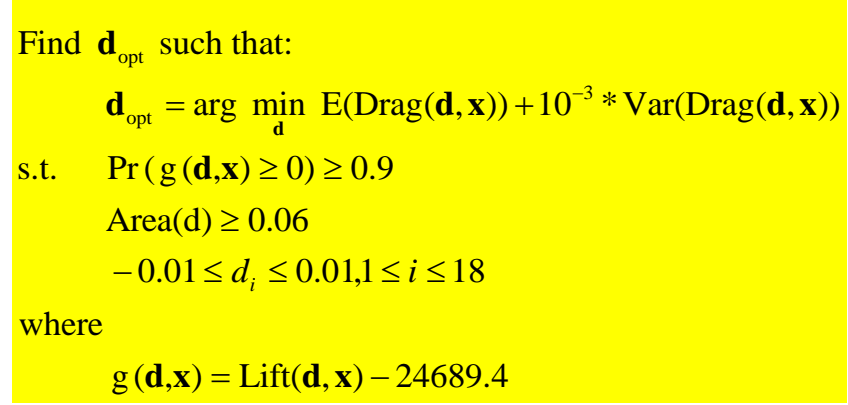

The initial design vector is defined as $d_{i}=0, i=1,2, \ldots, 20$. In the example, the flow field is discretized by a grid with 13550 elements. The grid around the airfoil and the contours of the pressure coefficient of the initial design are shown in Fig.17. The RAE2822 airfoil presents a strong upper-surface shock wave. In the optimization process, the gradients of drag and lift with respect to the design variables are calculated by the continuous adjoint method in the SU2 software (Economon et al. 2016; Yang et al. 2018).
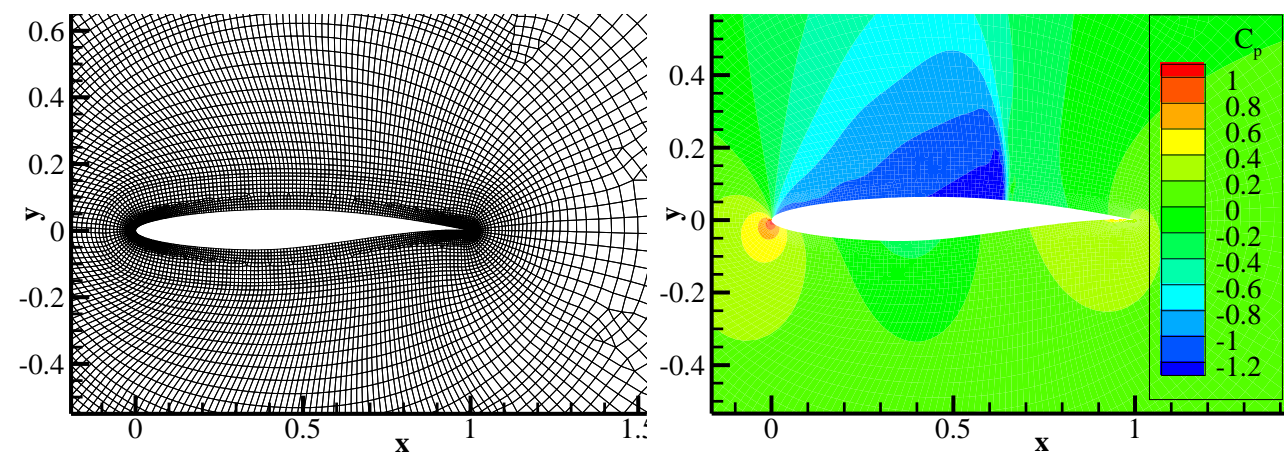

Fig. 17 Grid and pressure coefficient of the initial solution $\left(\mathrm{Ma}=7.29, \mathrm{AoA}=2^{\circ}\right)$

The index selection iteration steps for constructing the PCE of the airfoil problem are shown in Fig.18. During the iterations, the QOI for drag is the $\operatorname{Var}(\mathrm{Drag})$, and the QOI for the lift is $\operatorname{Pr}(\operatorname{Lift}>24689.4)$. For drag, the final error for $\operatorname{Var}\left(\mathrm{c}_{d}\right)$ is 1.64041 . For lift, the final error for $\operatorname{Pr}($ Lift $>24689.4)$ is $4.075 \times 10^{-4}$. The sample set containing 25 samples is used to construct the PCE of the lift and the drag.

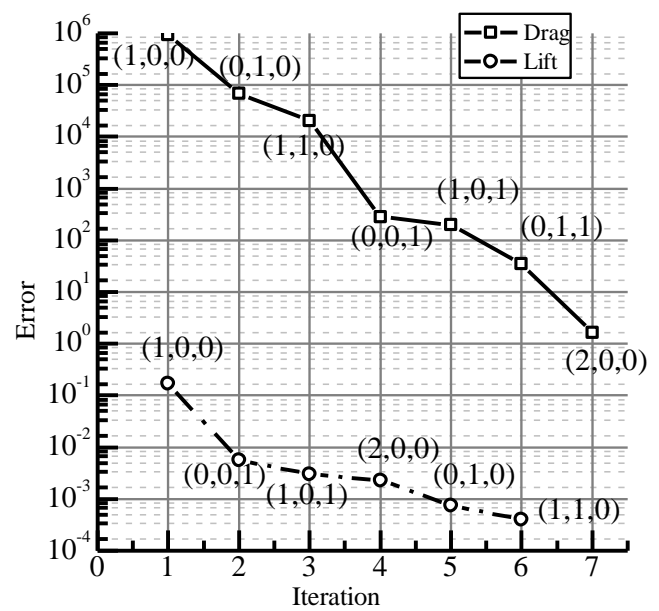

Fig.18 Airfoil optimization: index selection iteration steps 
The QOI of the initial and the optimal solutions are compared in Table 11. It is evident that both $\mathrm{E}\left(\mathrm{c}_{d}\right)$ and $\operatorname{Var}\left(\mathrm{c}_{d}\right)$ decrease while the $\operatorname{Pr}(\mathrm{g}(\mathbf{d}, \mathbf{x}) \geq 0)$ constraint is satisfied in the uncertainty optimization. The drag of the uncertain optimal solution has greater mean and variance than that of the deterministic optimal solution, which means that the optimization is the tradeoff between resistance and lift.

Table 11 Airfoil optimization: QOI comparison of the Initial, Optimal solutions

\begin{tabular}{|c|c|c|c|}
\hline & $\mathrm{E}($ Drag) $/(\mathrm{N})$ & $\operatorname{Var}($ Drag) /(N2) & $\operatorname{Pr}(\mathrm{Lift}>24689.4)$ \\
\hline Initial & 751.5433 & 906941.3411 & 0.89444 \\
\hline Deterministic Optimal & 335.3753 & 363384.5819 & 0.475843 \\
\hline Uncertainty Optimal & 494.7771 & 545454.4891 & 0.905447 \\
\hline
\end{tabular}

The contours of the pressure coefficient of the optimal solutions with $\mathrm{Ma}=7.29, \mathrm{AOA}=$ $2^{\circ}$ are shown in Fig.19 and Fig.20. The intensity of the shock wave on the upper surface is weaker compared to the initial design.
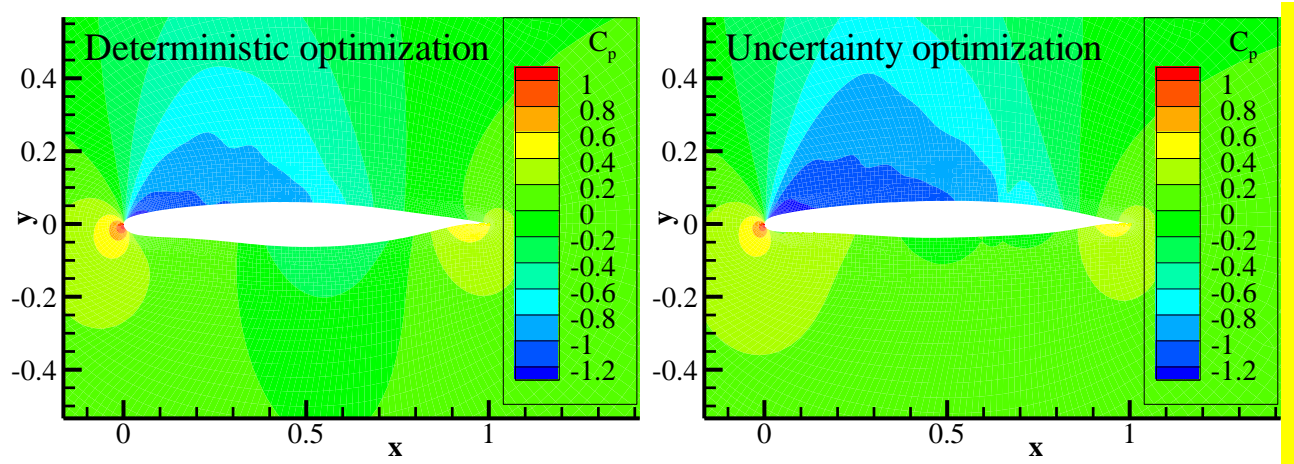

Fig.19 The pressure coefficient contours comparison $\left(\mathrm{Ma}=7.29, \mathrm{AOA}=2^{\circ}\right)$
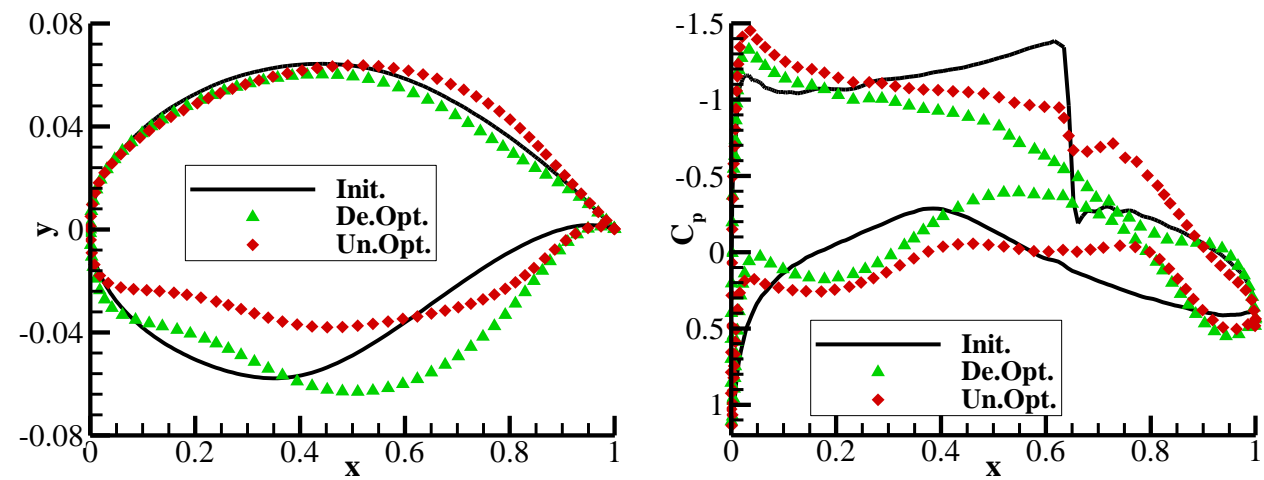

Fig.20 The airfoil surface pressure coefficient comparison $\left(\mathrm{Ma}=7.29, \mathrm{AOA}=2^{\circ}\right)$

Table 12 Airfoil optimization: geometric characteristics comparison

\begin{tabular}{|c|c|c|c|}
\hline & Leading edge radius & Maximum thickness & Maximum camber \\
\hline Initial & 0.008496 & 0.1211 & 0.00126 \\
\hline Deterministic Optimal & 0.00728 & 0.122829 & 0.005274 \\
\hline Uncertainty Optimal & 0.0073 & 0.101475 & 0.013667 \\
\hline
\end{tabular}

The geometric characteristics of the initial and optimal solutions are listed in Table 12. Compared with the initial design, the maximum camber angle of the uncertainty optimal solution increases naturally, which increases the lift to meet the reliability constraint. 

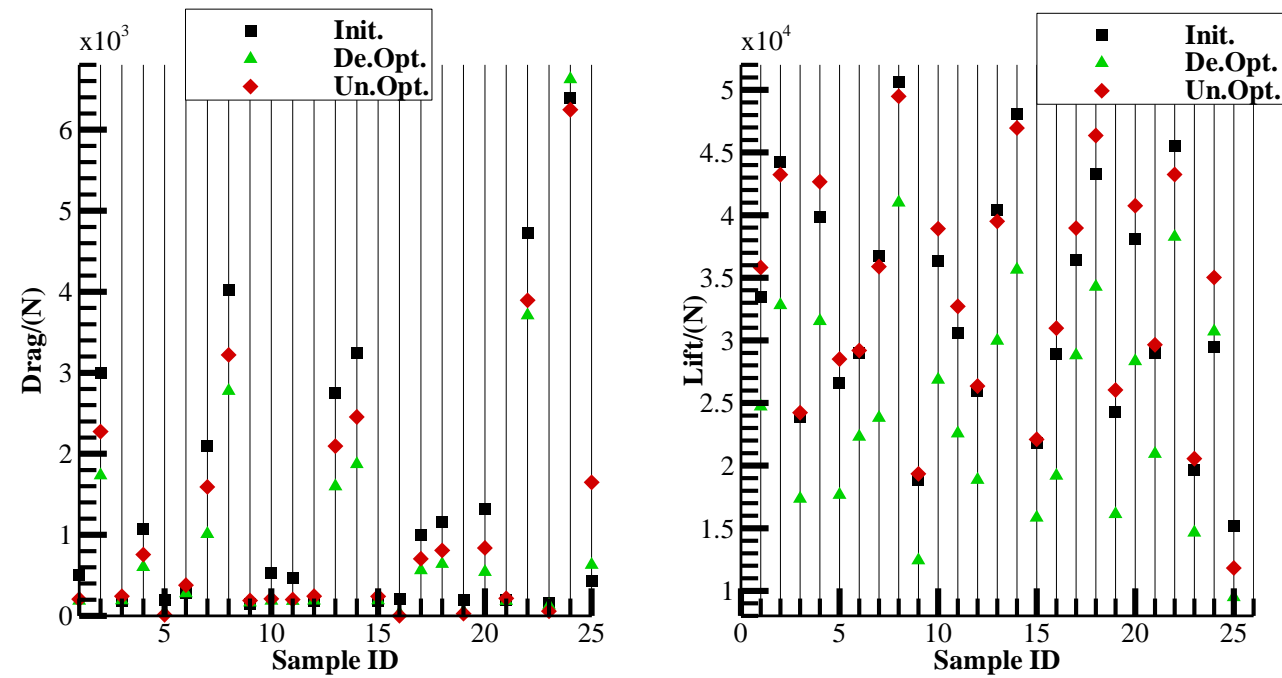

Fig.21 Airfoil optimization: sample set comparison

To further verify the optimization framework, the sample sets of the initial and the optimal solutions are compared in Fig.21. As is shown in Fig.21, the drag of the uncertainty optimization results are decreased, and the lift of the uncertainty optimization results are increased compared to the initial design for most of the samples.

\subsection{ONERA M6 Wing optimization}

The ONERA M6 wing optimization problem referenced from the literature (Palacios 2013) is modified to take into the aerodynamic uncertainty. The uncertainty parameters are listed in Table 13.

Table 13 ONERA M6 Wing optimization: freestream conditions

\begin{tabular}{|c|c|c|c|}
\hline & Distribution & Mean & Variance \\
\hline $\mathrm{V} /(\mathrm{m} / \mathrm{s})$ & Normal & 285.68 & 10 \\
\hline $\mathrm{AoA} /(\mathrm{o})$ & Normal & 3.06 & 0.5 \\
\hline Pressure/(Pa) & Normal & 101325.0 & 5066.25 \\
\hline
\end{tabular}

The geometry is parameterized using the free-form deformation (FFD) method. As shown in Fig.22, the solid black points represent the FFD control points of the FFD box, which are used to parameterize the ONERA M6 Wing. The z-axis coordinates of these FFD control points are the design variables. The movements of the control points on the $\mathrm{z}$-axis direction are defined as design variables within a given design range of $[-0.1,0.1]$.

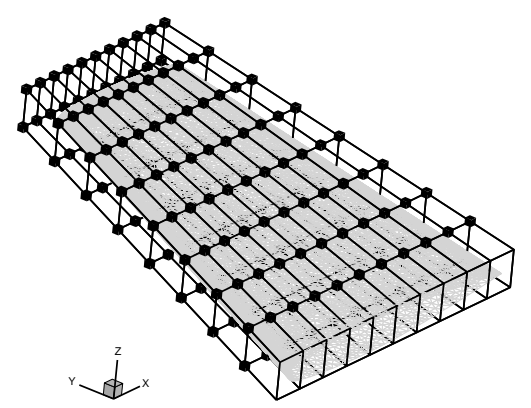

Fig.22 Parameterization of the ONERA M6 Wing

The uncertainty optimization problem defined in Eq.(45) is to minimize the drag while satisfying probabilistic constraints on the lift. The objective function is the sum of the drag mean value $\mathrm{E}($ Drag) and its variance $\operatorname{Var}(\operatorname{Drag})$. 


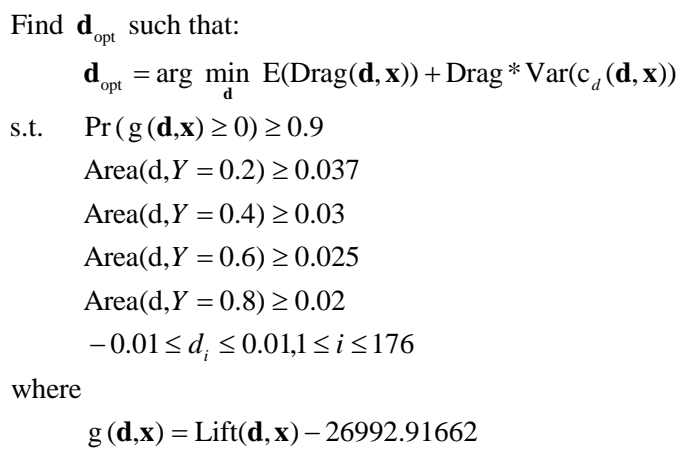

The initial design vector is assigned as $d_{i}=0, i=1,2, \ldots, 176$. In the example, the flow field is discretized by a grid with 582752 elements. The grid on the wing surface and the symmetry plane and the contours of the pressure coefficient of the initial design upper surface are shown in Fig.23. In the optimization process, the gradients of drag and lift with respect to the design variables are calculated by the continuous adjoint method in the SU2 software (Economon et al. 2016; Yang et al. 2018).

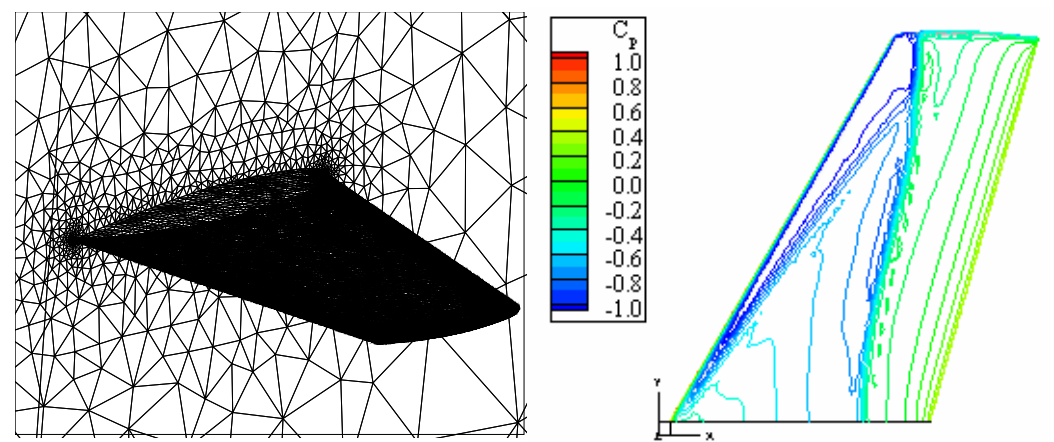

Fig.23 Grid and pressure coefficient of the initial solution $\left(\mathrm{V}=285.68 \mathrm{~m} / \mathrm{s}, \mathrm{AoA}=2^{\circ}\right.$, Pressure $=101325.0 \mathrm{~Pa}$ )

The index selection iteration steps for constructing the PCE of the wing problem are shown in Fig.24. During the iterations, the QOI for drag is the $\operatorname{Var}(\mathrm{Drag})$, and the QOI for the lift is $\operatorname{Pr}(\mathrm{Lift}>26992.91662)$. For drag, the final error for $\operatorname{Var}(\mathrm{Drag})$ is 48.63772. For lift, the final error for $\operatorname{Pr}(\mathrm{Lift}>26992.91662)$ is $7.13333 \times 10^{-5}$. The same sample containing 33 samples is used to construct PCE of lift and drag of the wing.

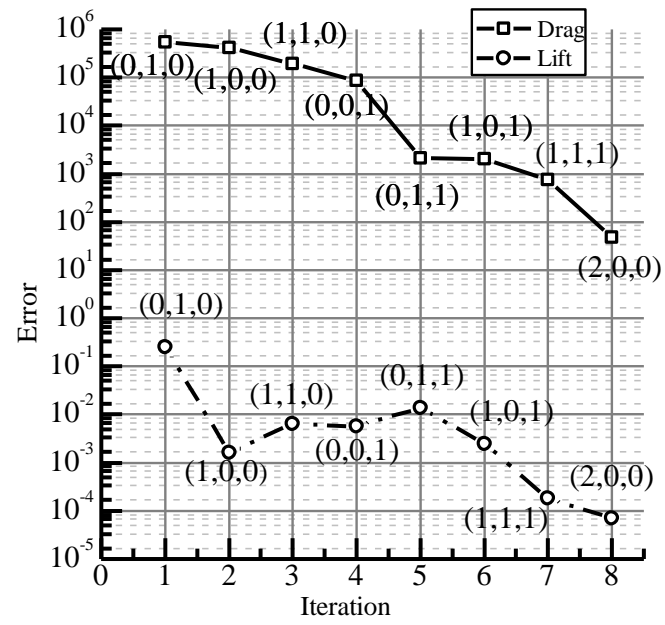

Fig.24 ONERA M6 Wing optimization: index selection iteration steps 
The QOIs of the initial and optimal solutions are compared in Table 14. Compared with the initial solution, both the lift and drag of the optimized solutions are improved. The uncertainty optimal solution has the largest increasing in lift to satisfy the reliability constraint, while the increasing of drag is also the largest. It clears that the optimization is a compromise between the lift performance and drag performance.

Table 14 ONERA M6 Wing optimization: QOI comparison of the Initial and Optimal solutions

\begin{tabular}{|c|c|c|c|}
\hline & $\mathrm{E}(\mathrm{Drag}) /(\mathrm{N})$ & $\operatorname{Var}(\mathrm{Drag}) /(\mathrm{N} 2)$ & $\operatorname{Pr}(\mathrm{Lift}>26992.91662)$ \\
\hline Initial & 770.891312 & 288978.0503 & 0.002785 \\
\hline $\begin{array}{c}\text { Uncertainty } \\
\text { optimization }\end{array}$ & 3262.808868 & 1148540.468 & 0.904385 \\
\hline $\begin{array}{c}\text { Deterministic } \\
\text { optimization }\end{array}$ & 1911.880519 & 653316.417 & 0.496382 \\
\hline
\end{tabular}

The deformation of the FFD box and the wing are shown in Fig.25. As can be seen, the camber of the wing airfoil has increased in both the deterministic and uncertainty optimization. The increase in the wing airfoil increases the lift of the wing.

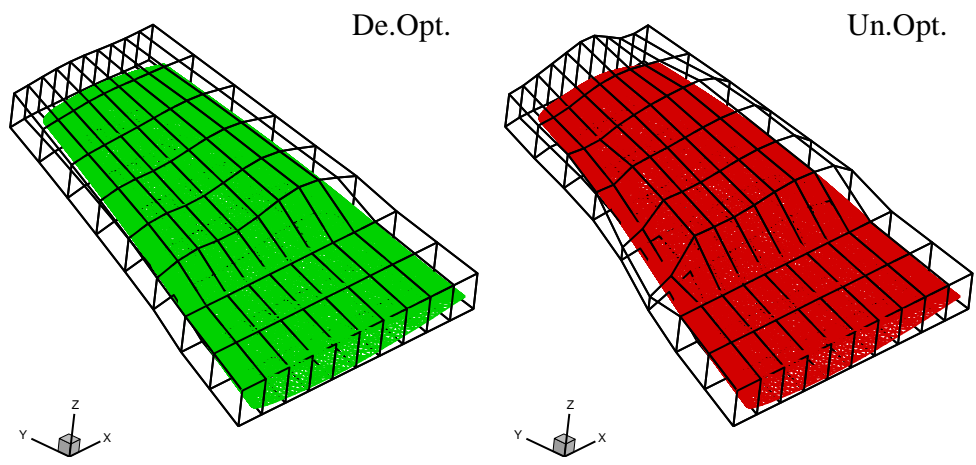

Fig.25 FFD box and the wing deformation

The contours of the pressure coefficient on the upper surface of the optimal solutions with $\mathrm{V}=285.68 \mathrm{~m} / \mathrm{s}, \mathrm{AoA}=2^{\circ}$, Pressure $=101325.0 \mathrm{~Pa}$ are shown in Fig.26 and Fig.27. Compared with the initial solution, the thickness and camber of the wing slices of the optimized solution are all improved. The uncertainty optimal solution has even larger increasing which results in lager lift and drag.
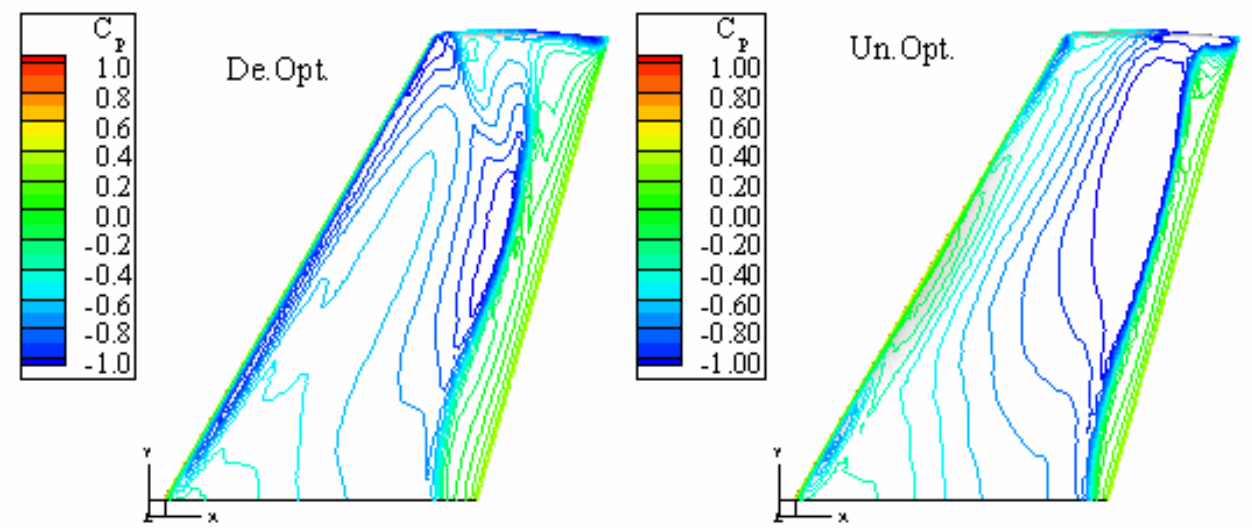

Fig.26 Pressure coefficient of the optimal solutions $\left(\mathrm{V}=285.68 \mathrm{~m} / \mathrm{s}, \mathrm{AoA}=2^{\circ}\right.$, Pressure $\left.=101325.0 \mathrm{~Pa}\right)$ 

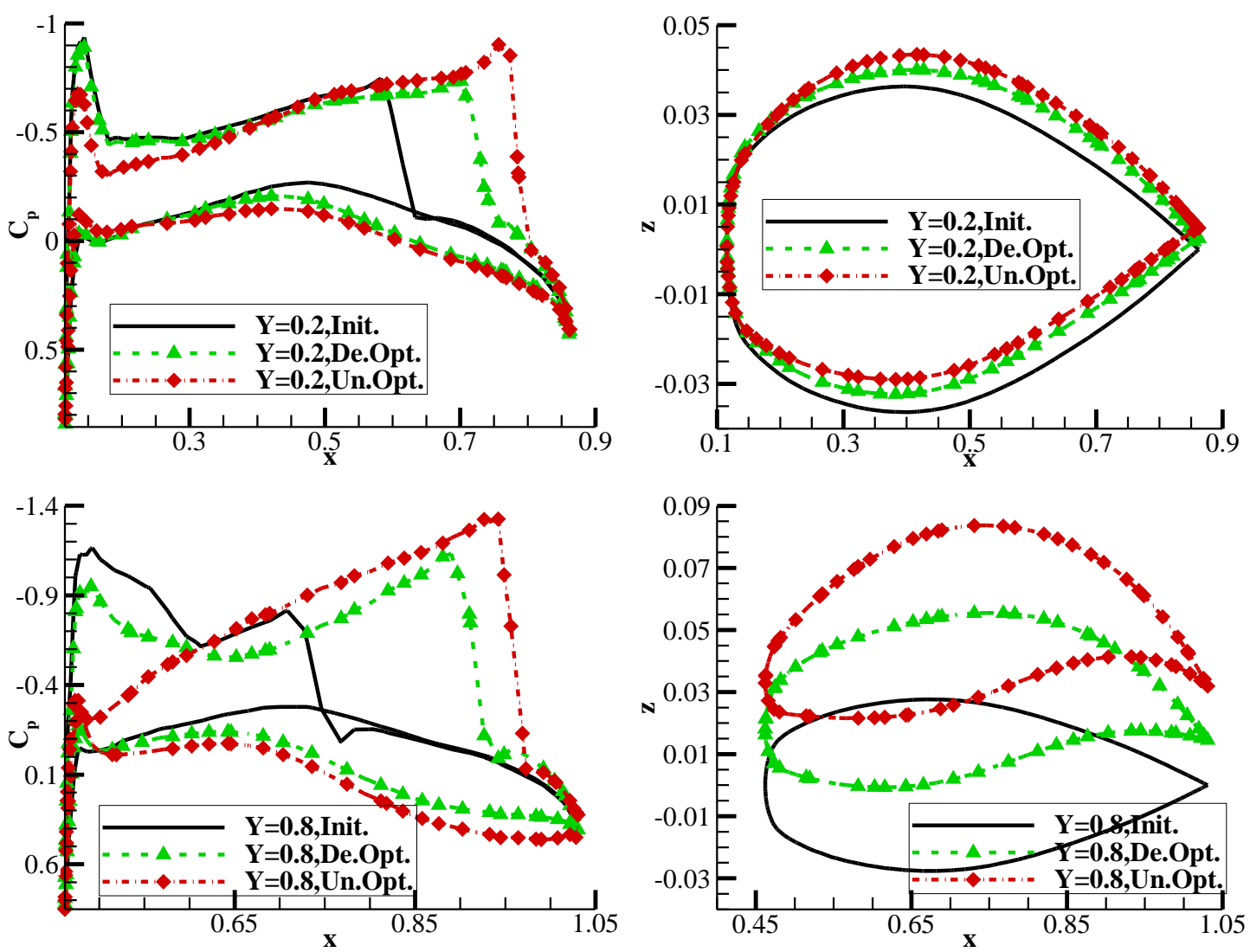

Fig.27 Pressure coefficient and airfoils of the wing slices $\left(V=285.68 \mathrm{~m} / \mathrm{s}, \mathrm{AoA}=2^{\circ}\right.$, Pressure $=101325.0$ )
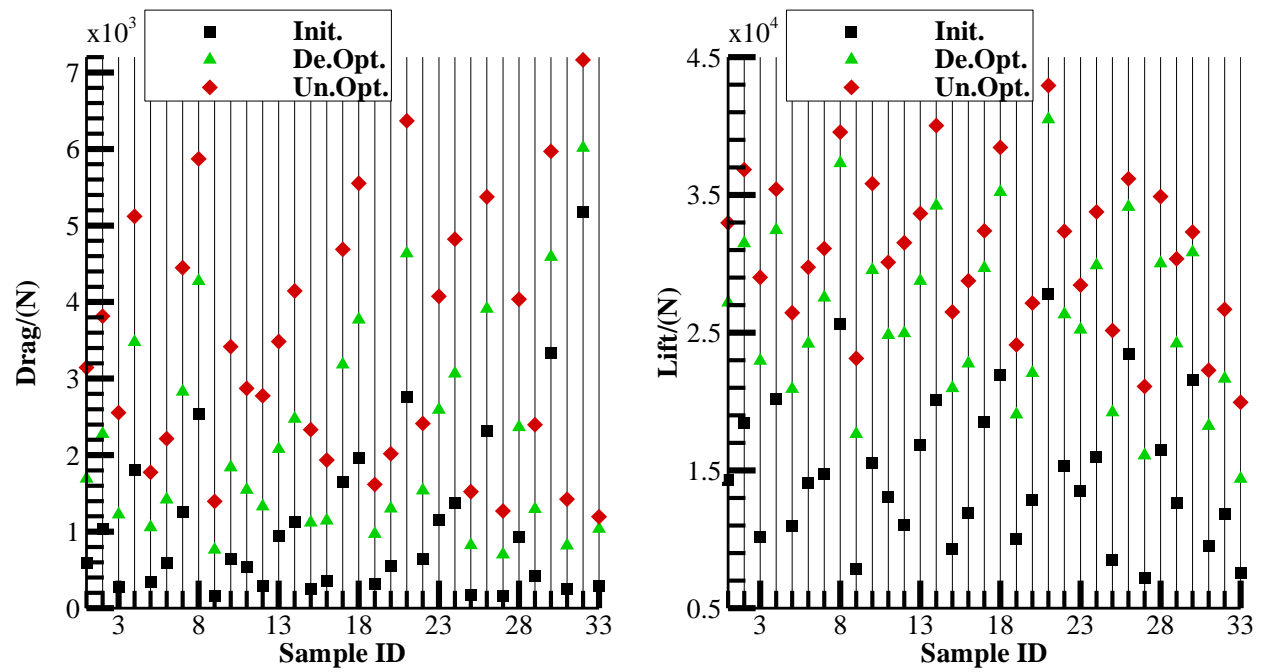

Fig.28 ONERA M6 Wing optimization: sample set comparison

To further verify the optimization, the sample sets of the initial and optimal solutions are compared in Fig.28. As shown in Fig.28, both the lift and the drag of the uncertainty optimization results are the largest among the solutions. 


\section{Conclusion}

To solve the design optimization problem based on robustness and reliability using gradient-based algorithms, a framework is presented with the PCE to evaluate the statistical indicators and their sensitivity. The PCE coefficients are evaluated by the dimensional adaptive sparse grid, which relieves the computational burden incurred by the curse of dimensionality. The statistical moments and their gradients are derived directly from the PCE. The reliability is obtained by the integration of the Heaviside step function with the MCS. The reliability sensitivity is derived by combining the PCE derivative, Taylor's expansion, and the finite difference. The optimization framework is formed by combining reliability, robustness quantification, sensitivity analysis and the optimization module.

The accuracy and efficiency of reliability quantification and the reliability sensitivity analysis are verified through a mathematical example, a system of springs, and a cantilever beam. For the mathematical example and the system of springs, by comparing the proposed method with the FORM, the SORM, the PDEM, and a method available from the literature, it was found that the proposed method could obtain the same level of accuracy as directly using the MCS with but fewer function evaluations. The cantilever tube example shows that the framework can identify the linearity and non-linearity of the functions, and the proposed criterion is effective. The effectiveness of the framework used in several optimization problems is verified through a cantilever beam optimization problem, a multiple limit states example, a truss optimization example, an airfoil problem, and an ONERA M6 wing optimization problem. In the cantilever beam optimization problem and the multiple limit states example, the comparison between the proposed framework and the method from the literature shows that the proposed framework has the advantage in accuracy and efficiency in solving the uncertainty optimization problem. In the truss optimization example, an airfoil problem, and an ONERA M6 wing optimization, the results show the effectiveness of the proposed framework in solving the engineering uncertainty optimization problem. The framework has the shortage inherited from the gradient optimization algorithm, which may converge with the local minimum solution. The ability of the proposed framework reduces the computation burden of using a gradient-based algorithm to solve uncertainty optimization problems.

\section{Acknowledgment}

The present work was partially supported by the Fundamental Research Funds for the Central Universities (Grant No. G2016KY0302), the National Defense Fundamental Research Funds of China (Grant No. JCKY2016204B102), and China Civil Aerospace Program (Grant No. D010403), the Natural National Science Foundation of China (Grant No. 51505385, 11502209).

\section{References}

Ahammed M, Melchers RE (2006) Gradient and parameter sensitivity estimation for systems evaluated using Monte Carlo analysis Reliability Engineering \& System Safety 91:594-601 doi:10.1016/j.ress.2005.04.005

Au SK (2005) Reliability-based design sensitivity by efficient simulation Computers \& Structures 83:1048-1061 doi:10.1016/j.compstruc.2004.11.015

Beyer HG, Sendhoff B (2007) Robust optimization - A comprehensive survey Computer Methods in Applied Mechanics and Engineering 196:3190-3218 doi:10.1016/j.cma.2007.03.003

Bungartz HJ, Dirnstorfer S (2003) Multivariate quadrature on adaptive sparse grids Computing 71:89-114 doi:10.1007/s00607-003-0016-4

Ching JY, Hsieh YH (2007) Local estimation of failure probability function and its confidence interval with maximum entropy principle Probabilistic Engineering Mechanics 22:39-49 doi:10.1016/j.probengmech.2006.05.002

Da Ronch A, Ghoreyshi M, Badcock KJ (2011) On the generation of flight dynamics aerodynamic 
tables by computational fluid dynamics Prog Aeosp Sci 47:597-620 doi:10.1016/j.paerosci.2011.09.001

Ditlevsen O, Bjerager P (1989) Plastic Reliability-Analysis by Directional Simulation Journal of Engineering Mechanics-Asce doi:10.1061/(Asce)0733-9399(1989)115:6(1347)

Du S, Guo J, Zhao Z, Zeng S Non-intrusive polynomial chaos based mechanism reliability sensitivity analysis. In: 2014 Prognostics and System Health Management Conference (PHM-2014 Hunan), 24-27 Aug. 2014 2014. pp 515-519. doi:10.1109/PHM.2014.6988226

Du XP, Chen W (2004) Sequential optimization and reliability assessment method for efficient probabilistic design Journal of Mechanical Design 126:225-233 doi:10.1115/1.1649968

Duan NQ (2006) Optimize Truss by FSD and SLP doi:https://ww2.mathworks.cn/matlabcentral/fileexchange/12401-optimize-truss-by-fsd-and-s Ip (online available)

Economon TD, Palacios F, Copeland SR, Lukaczyk TW, Alonso JJ (2016) SU2: An Open-Source Suite for Multiphysics Simulation and Design AIAA Journal 54:828-846 doi: $10.2514 / 1 . j 053813$

Eldred M, Burkardt J (2009) Comparison of Non-Intrusive Polynomial Chaos and Stochastic Collocation Methods for Uncertainty Quantification. In: 47th AIAA Aerospace Sciences Meeting including The New Horizons Forum and Aerospace Exposition. Aerospace Sciences Meetings. American Institute of Aeronautics and Astronautics. doi:10.2514/6.2009-976

Eldred MS, Agarwal H, Perez VM, Wojtkiewicz SF, Renaud JE (2007) Investigation of reliability method formulations in DAKOTA/UQ Structure and Infrastructure Engineering 3:199-213 doi:10.1080/15732470500254618

Enevoldsen I, Sørensen JD (1993) Reliability-Based Optimization of Series Systems of Parallel Systems Journal of Structural Engineering 119:1069-1084 doi:10.1061/(asce)0733-9445(1993)119:4(1069)

Garcke J, Griebel M, Thess M (2001) Data mining with sparse grids Computing 67:225-253 doi: $10.1007 / \mathrm{s} 006070170007$

Gerstner T, Griebel M (2003) Dimension-adaptive tensor-product quadrature Computing 71:65-87 doi:10.1007/s00607-003-0015-5

Hohenbichler M, Rackwitz R (1986) Sensitivity and importance measures in structural reliability Civil Engineering Systems 3:203-209 doi:10.1080/02630258608970445

Hu XZ, Chen XQ, Parks GT, Yao W (2016) Review of improved Monte Carlo methods in uncertainty-based design optimization for aerospace vehicles Prog Aeosp Sci 86:20-27 doi:10.1016/j.paerosci.2016.07.004

Jensen HA, Mayorga F, Valdebenito MA (2015) Reliability sensitivity estimation of nonlinear structural systems under stochastic excitation: A simulation-based approach Computer Methods in Applied Mechanics and Engineering 289:1-23 doi:10.1016/j.cma.2015.01.012

Karamchandani A, Cornell CA (1992) Sensitivity estimation within first and second order reliability methods Structural Safety 11:95-107 doi:10.1016/0167-4730(92)90002-5

Keshavarzzadeh V, Fernandez F, Tortorelli DA (2017) Topology optimization under uncertainty via non-intrusive polynomial chaos expansion Computer Methods in Applied Mechanics and Engineering 318:120-147 doi:10.1016/j.cma.2017.01.019

Keshavarzzadeh V, Meidani H, Tortorelli DA (2016) Gradient based design optimization under uncertainty via stochastic expansion methods Computer Methods in Applied Mechanics and Engineering 306:47-76 doi:10.1016/j.cma.2016.03.046

Keshtegar B, Hao P (2016) A Hybrid Loop Approach Using the Sufficient Descent Condition for Accurate, Robust, and Efficient Reliability-Based Design Optimization Journal of Mechanical Design 138:121401-121401-121411 doi:10.1115/1.4034173

Keshtegar B, Hao P (2017) A hybrid self-adjusted mean value method for reliability-based design optimization using sufficient descent condition Applied Mathematical Modelling 41:257-270 doi:https://doi.org/10.1016/j.apm.2016.08.031

Keshtegar B, Hao P (2018) Enhanced single-loop method for efficient reliability-based design optimization with complex constraints Structural and Multidisciplinary Optimization 57:1731-1747 doi:10.1007/s00158-017-1842-x

Klimke WA (2006) Uncertainty modeling using fuzzy arithmetic and sparse grids. Universita“t Stuttgart 
Kwak BM, Lee TW (1987) Sensitivity Analysis for Reliability-Based Optimization Using an Afosm Method Computers \& Structures 27:399-406 doi:10.1016/0045-7949(87)90064-2

Lelievre N, Beaurepaire P, Mattrand C, Gayton N, Otsmane A (2016) On the consideration of uncertainty in design: optimization - reliability - robustness Structural and Multidisciplinary Optimization 54:1423-1437 doi:10.1007/s00158-016-1556-5

Li J (2016) Probability density evolution method: Background, significance and recent developments Probabilistic Engineering Mechanics 44:111-117 doi:https://doi.org/10.1016/j.probengmech.2015.09.013

Lopez RH, Beck AT (2012) Reliability-Based Design Optimization Strategies Based on FORM: A Review Journal of the Brazilian Society of Mechanical Sciences and Engineering 34:506-514 doi:10.1590/S1678-58782012000400012

Melchers RE, Ahammed M (2004) A fast approximate method for parameter sensitivity estimation in Monte Carlo structural reliability Computers \& Structures 82:55-61 doi:10.1016/j.compstruc.2003.08.003

Meng Z, Li G, Wang BP, Hao P (2015) A hybrid chaos control approach of the performance measure functions for reliability-based design optimization Computers \& Structures 146:32-43 doi:https://doi.org/10.1016/j.compstruc.2014.08.011

Meng Z, Zhou H, Li G, Yang D (2016) A decoupled approach for non-probabilistic reliability-based design optimization Computers \& Structures 175:65-73 doi:https://doi.org/10.1016/j.compstruc.2016.06.008

Meza JC, Oliva RA, Hough PD, Williams PJ (2007) OPT++: An object-oriented toolkit for nonlinear optimization Acm Transactions on Mathematical Software 33:12 doi:10.1145/1236463.1236467

Padulo M, Campobasso MS, Guenov MD (2011) Novel Uncertainty Propagation Method for Robust Aerodynamic Design Aiaa Journal 49:530-543 doi:10.2514/1.J050448

Palacios F (2013) Problem Workshop II: Design and Optimization Using SU2 doi:https://su2code.github.io/documents/Design and Optimization_Using_SU2.pdf (online available)

Papadimitriou DI, Papadimitriou C (2016) Aerodynamic shape optimization for minimum robust drag and lift reliability constraint Aerospace Science and Technology 55:24-33 doi:10.1016/j.ast.2016.05.005

Park C, Kim NH, Haftka RT (2015) The effect of ignoring dependence between failure modes on evaluating system reliability Structural and Multidisciplinary Optimization 52:251-268 doi:10.1007/s00158-015-1239-7

Rackwitz R (2001) Reliability analysis-a review and some perspectives Structural Safety 23:365-395 doi:10.1016/s0167-4730(02)00009-7

Royset JO, Polak E (2004) Reliability-based optimal design using sample average approximations Probabilistic Engineering Mechanics 19:331-343 doi:10.1016/j.probengmech.2004.03.001

Schillings C (2010) Optimal Aerodynamic Design under Uncertainties. Universität Trier

Schillings C, Schulz V (2015) On the influence of robustness measures on shape optimization with stochastic uncertainties Optimization and Engineering 16:347-386 doi:10.1007/s11081-014-9251-0

Smoljak SA (1963) Quadrature and interpolation formulas for tensor products of certain classes of $\begin{array}{lllll}\text { functions } & \text { Dokl } & \text { Akad } & \text { Nauk } & \text { SSSR } \\ \text { 148:1042-1045 }\end{array}$ doi:http://www.mathnet.ru/php/archive.phtml?wshow=paper\&jrnid=dan\&paperid=27586\&op tion lang=eng (online available)

Taflanidis AA, Jia GF (2011) A simulation-based framework for risk assessment and probabilistic sensitivity analysis of base-isolated structures Earthquake Engineering \& Structural Dynamics 40:1629-1651 doi:10.1002/eqe.1113

Torii AJ, Lopez RH, Miguel LFF (2017) A gradient-based polynomial chaos approach for risk and reliability-based design optimization Journal of the Brazilian Society of Mechanical Sciences and Engineering 39:2905-2915 doi:10.1007/s40430-017-0815-8

Valdebenito MA, Jensen HA, Hernandez HB, Mehrez L (2018) Sensitivity estimation of failure probability applying line sampling Reliability Engineering \& System Safety 171:99-111 doi:10.1016/j.ress.2017.11.010

Verma AK, Ajit S, Karanki DR (2016) Reliability and Safety Engineering. Springer London, London. doi:10.1007/978-1-4471-6269-8 
Wang HY, Kim NH, Kim YJ (2006) Safety envelope for load tolerance and its application to fatigue reliability design Journal of Mechanical Design 128:919-927 doi:10.1115/1.2204971

Wu X, Zhang W, Song S (2018) Robust aerodynamic shape design based on an adaptive stochastic optimization framework Structural and Multidisciplinary Optimization 57:639-651 doi:10.1007/s00158-017-1766-5

Wu YT (1994) Computational Methods for Efficient Structural Reliability and Reliability Sensitivity Analysis Aiaa Journal 32:1717-1723 doi:10.2514/3.12164

Xiu DB, Karniadakis GE (2002) The Wiener-Askey polynomial chaos for stochastic differential equations Siam Journal on Scientific Computing 24:619-644 doi:10.1137/S1064827501387826

Yanfang Z, Yanlin Z, Yimin Z (2011) Reliability sensitivity based on first-order reliability method vol 225. doi:10.1177/0954406211405938

Yang D, Liu L (2014) Reliability analysis of structures with complex limit state functions using probability density evolution method Structural and Multidisciplinary Optimization 50:275-286 doi:10.1007/s00158-014-1048-4

Yang G, Da Ronch A, Drofelnik J, Xie Z-T (2018) Sensitivity assessment of optimal solution in aerodynamic design optimisation using SU2 Aerospace Science and Technology 81:362-374 doi:https://doi.org/10.1016/j.ast.2018.08.012

Yao W, Chen XQ, Luo WC, van Tooren M, Guo J (2011) Review of uncertainty-based multidisciplinary design optimization methods for aerospace vehicles Prog Aeosp Sci 47:450-479 doi:10.1016/j.paerosci.2011.05.001

Yi P, Cheng GD, Jiang L (2008) A sequential approximate programming strategy for performance-measure-based probabilistic structural design optimization Structural Safety 30:91-109 doi:10.1016/j.strusafe.2006.08.003

Youn BD, Choi KK, Du L (2005) Enriched Performance Measure Approach for Reliability-Based Design Optimization AIAA Journal 43:874-884 doi:10.2514/1.6648

Zhang T (2018) Reliability-Based Robust Optimal Design of Structures with Variable Cross-Section vol 55. doi:10.1007/978-981-10-6553-8_111

Zhang T, Zhang Y (2016) A new model for reliability design and reliability sensitivity analysis of a hydraulic piston pump Proceedings of the Institution of Mechanical Engineers, Part O: Journal of Risk and Reliability 231:11-24 doi:10.1177/1748006x16681713

Zhao W, Liu JK, Ye JJ (2011) A new method for parameter sensitivity estimation in structural reliability analysis Applied Mathematics and Computation 217:5298-5306 doi:10.1016/j.amc.2010.11.053 